\title{
Feasibility of Batch Reactive Distillation with Equilibrium-Limited Consecutive Reactions in Rectifier, Stripper, or Middle-Vessel Column
}

\author{
T. Lukács, ${ }^{1}$ C. Stéger, ${ }^{1}$ E. Rév, ${ }^{1}$ M. Meyer, ${ }^{2}$ and Z. Lelkes ${ }^{1}$ \\ ${ }^{1}$ Department of Chemical and Environmental Process Engineering, Budapest Univesity of Technology and Economics, \\ Muegyetem rkp. 3, 1521 Budapest, Hungary \\ ${ }^{2}$ Laboratoire de Génie Chimique, Ecole Nationale Supérieure d’Ingénieurs en Arts Chimiques et Technologiques, UMR CNRS 5503, \\ ENSIACET-UPS-CNRS, BP 1301, 5 rue Paulin Talabot, 31106 Toulouse, France
}

Correspondence should be addressed to T. Lukács, timcsilincsi@gmail.com

Received 7 March 2011; Accepted 1 August 2011

Academic Editor: Raghunath V. Chaudhari

Copyright (๑) 2011 T. Lukács et al. This is an open access article distributed under the Creative Commons Attribution License, which permits unrestricted use, distribution, and reproduction in any medium, provided the original work is properly cited.

\begin{abstract}
A general overall feasibility methodology of batch reactive distillation of multireaction systems is developed to study all the possible configurations of batch reactive distillation. The general model equations are derived for multireaction system with any number of chemical equilibrium-limited reactions and for any number of components. The present methodology is demonstrated with the detailed study of the transesterification of dimethyl carbonate in two reversible cascade reactions in batch reactive distillation process. Pure methanol is produced as distillate, and pure diethyl carbonate is produced at the bottom simultaneously in middlevessel column; in each section, continuous feeding of ethanol is necessary. The results of feasibility study are successfully validated by rigorous simulations.
\end{abstract}

\section{Introduction}

Reactive distillation is a hybrid process integrating separation and reaction in a single unit. The main advantages of reactive distillation compared to the sequential processes include reduced investment and operating costs, reduced utility consumption, and higher conversion and selectivity [1]. Nevertheless, reactive distillation is not advantageous in every case, and systematic methods are needed to examine its feasibility for particular reaction systems.

Design of complex processes usually starts with feasibility analysis including computation of limiting flows and minimum stage numbers as well. Quick, simple, and reliable method is needed to find the best feasible configurations and parameter regions applicable to produce the desired products.

Several methodologies for the preliminary study of reactive distillation have been published in the past decades; short overview is in Stéger et al. [2]. Many of them are based on graphical techniques providing a basis for developing conceptual designs (e.g., in the articles of [3-6]). In spite of the efforts, their application is still limited because of the lack of a systematic design method applicable in general case, especially for complex column configurations [2,7].

All the possible configurations of batch reactive distillation with equilibrium-limited single reactions in rectifier with reactive boiler can be analyzed with the reliable, overall feasibility method suggested by Stéger et al. [2]. Only configurations with at most one feed are discussed there but the method can be extended for several entrainer feeds [7].

In the present work, we extend our general feasibility method for any number of equilibrium-limited reactions in middle-vessel column, stripper, and rectifier. The version extended for any number of reactions and components of general model equation presented in Stéger et al. [2] for the reactive zone is derived here. Middle-vessel column, its upper part as a rectifier, and the lower part as a stripper are all included in the analysis.

Multireaction system containing two or more reactions and multicomponent systems containing four or more 
components have already been published in the literature, but none of them deals with their overall feasibility analysis for batch processes, examining all the possible configuration.

In the present work, we present the methodology of an overall feasibility study of any multicomponent and multireaction system for batch processes and any configuration of middle vessel column, stripper, and rectifier. The presented methodology can also be used to continuous processes after applying some modifications [8].

The method is demonstrated in studying the feasibility of transesterification of dimethyl carbonate (DMC) in two cascade reversible reactions among five components with an intermediate methyl ethyl carbonate (MEC) [9] in batch reactive distillation procedure. Although modeling the production of DEC in a continuous reactive distillation process has been published by Luo and Xiao [9], its overall feasibility study has not yet been published.

\section{The Feasibility Method}

2.1. Single-Reaction Systems. During the feasibility study, a simplified model is used with the common assumptions like instantaneous equilibrium-limited reaction in the liquid phase, neglected reaction enthalpy, and liquid hold-up on the stages, instantaneous vapor-liquid equilibrium with neglected catalyst effect [2]. Due to the simplifying assumptions, the feasible configurations can be found in a short time, and the feasible domain of the main operation parameters can also be determined.

Batch extractive distillation columns can be divided into three different zones: rectifying or stripping section, extractive section, and still vessel [10-12]. The rectifying section is located below the distillate and the stripping section above the bottom product. Any extractive section is located between the middle-vessel and some feed. This terminology is applicable to batch rectifier, stripper, and middle vessel column as is shown in Figure 1. Applying heterogeneous catalyst, any section can be either reactive or nonreactive. Whereas, applying homogeneous catalyst, the sections below the catalyst feed must be reactive.

There are several possible configurations of batch reactive distillation in a batch reactive rectifier or in a batch reactive stripper considering a reactive vessel and at most one feed, depending on the place of the reaction and feed locations. For example, a batch rectifier with at most one entrainer feed and a single contiguous reactive section including the still may consist of the still and the following alternatives listed bottom up in each version (1) a nonreactive rectifying section, (2) a reactive rectifying section, (3) a reactive rectifying section and a nonreactive rectifying section, (4) a nonreactive extractive section below an entrainer feed, and a nonreactive rectifying section, (5) a reactive extractive section below an entrainer feed, and a reactive rectifying section, (6) a reactive extractive section below an entrainer feed, and a nonreactive rectifying section, (7) a reactive extractive section below an entrainer feed, a reactive rectifying section and a nonreactive rectifying section, or (8) a reactive extractive section below a nonreactive extractive section below an entrainer feed, and

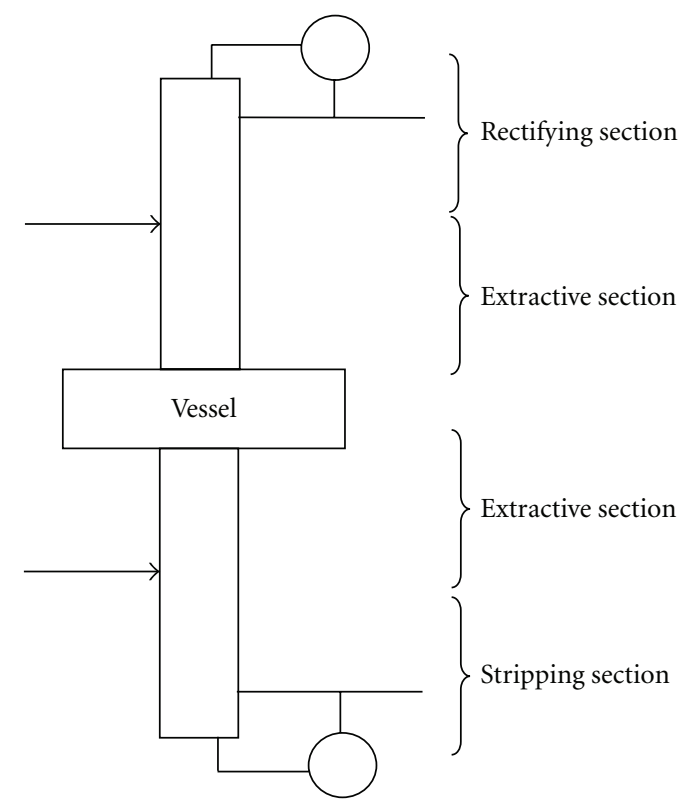

FIGURE 1: Middle vessel column.

a nonreactive rectifying section. Locating the feed directly to the top of the column or to the still vessel [13] is not even considered in the list above. There may be more feeds; however, and the number of contiguous reactive sections can also be higher if heterogeneous catalysis is applied. Thus, a large number of configurations can be conceived for a middlevessel column because it is a combination of a rectifier and a stripper. One aim of the preliminary study is to select the feasible configurations.

The methodology published in Stéger et al. [2] is further developed here to cope with this combinatorial diversity. Instead of considering all the configurations separately, the possible composition profiles in the potential column sections are analyzed in the proposed feasibility study. No column length constraint is taken into account, namely, infinite length is considered. The ensemble of possible profiles is constrained by product purity specifications and by the charge composition.

Due to assuming instantaneous equilibrium-limited reactions, the column profiles can be represented in a transformed space. With the transformation,

$$
\tilde{a}_{i}=a_{i}-\frac{v_{i}}{v_{\mathrm{ref}}} a_{\mathrm{ref}}
$$

proposed by Stéger et al. [2], where " $a_{i}$ " can be any liquid, vapor, feed, or product component flow rate, the reactive profiles are brought to the same form used for the nonreactive sections by Lelkes et al. [10] and Varga et al. [12] (Table 1). 
TABLE 1: Model equations of column sections for single-reaction systems.

\begin{tabular}{|c|c|c|}
\hline Section & Nonreactive & Reactive \\
\hline Rectifying & $\begin{array}{l}\frac{d x_{i}}{d h}=\left(\frac{V}{L}\right)\left(y_{i}-y_{i}^{*}\right) \text { where } \\
y_{i}=\left(\frac{L}{V}\right) x_{i}+\left(\frac{D}{V}\right) x_{D, i}[13]\end{array}$ & $\frac{d \tilde{l}_{i}}{d h}=\tilde{v}_{i}-\tilde{v}_{i}^{*}$ where $\tilde{v}_{i}=\tilde{l}_{i}+\tilde{d}_{i}[2]$ \\
\hline Extractive (in a rectifier) & $\begin{array}{l}\frac{d x_{i}}{d h}=\left(\frac{L^{\prime}}{V}\right)\left(y_{i}-y_{i}^{*}\right) \text { where } \\
y_{i}=\left(\frac{L^{\prime}}{V}\right) x_{i}+\left(\frac{D}{V}\right) x_{D, i}-\left(\frac{F_{d}}{V}\right) x_{f d, i}[13]\end{array}$ & $\frac{d \tilde{l}_{i}^{\prime}}{d h}=\tilde{v}_{i}-\tilde{v}_{i}^{*}$ where $\tilde{v}_{i}=\tilde{l}_{i}^{\prime}+\tilde{d}_{i}-\tilde{f}_{d, i}[2]$ \\
\hline Stripping & $\begin{array}{l}\frac{d x_{i}}{d h}=\left(\frac{V}{L}\right)\left(y_{i}-y_{i}^{*}\right) \text { where } \\
y_{i}=\left(\frac{L}{V}\right) x_{i}-\left(\frac{W}{V}\right) x_{W, i}[11]\end{array}$ & $\frac{d \tilde{l}_{i}}{d h}=\tilde{v}_{i}-\tilde{v}_{i}^{*}$ where $\tilde{v}_{i}=\tilde{l}_{i}-\tilde{w}_{i}[2]$ \\
\hline Extractive (in a stripper) & $\begin{array}{l}\frac{d x_{i}}{d h}=\left(\frac{L^{\prime}}{V}\right)\left(y_{i}-y_{i}^{*}\right) \text { where } \\
y_{i}=\left(\frac{V}{L^{\prime}}\right) x_{i}-\left(\frac{W}{V}\right) x_{W, i}+\left(\frac{F_{w}}{V}\right) x_{f w, i}[11]\end{array}$ & $\frac{d \tilde{l}_{i}^{\prime}}{d h}=\tilde{v}_{i}-\tilde{v}_{i}^{*}$ where $\tilde{v}_{i}=\tilde{l}_{i}^{\prime}-\tilde{w}_{i}+\tilde{f}_{w, i}[2]$ \\
\hline
\end{tabular}

TABLE 2: Model equations of vessel path for single-reaction systems.

\begin{tabular}{|c|c|}
\hline Nonreactive & $\frac{d x_{i}}{d t}=D\left(x_{i}-x_{D, i}\right)+W\left(x_{i}-x_{W, i}\right)-F_{d}\left(x_{f d, i}-x_{i}\right)-F_{w}\left(x_{f w, i}-x_{i}\right)$ \\
\hline Reactive, $\nu_{T}=0$ & $\begin{array}{l}\frac{a X_{i}}{d t}=D\left(X_{i}-X_{D, i}\right)+W\left(X_{i}-X_{W, i}\right)-F_{d}\left(X_{f d, i}-X_{i}\right)-F_{w}\left(X_{f w, i}-X_{i}\right) \\
\tilde{U}=U\left(1-\left(\frac{v_{T}}{\nu_{\text {ref }}}\right) x_{\text {ref }}\right) \text { and }\end{array}$ \\
\hline Reactive, $v_{T} \neq 0$ & $\begin{array}{l}\tilde{U}\left(\frac{d X_{i}}{d t}\right)=\widetilde{D} \cdot\left(X_{i} \cdot \frac{\left(1-\left(\nu_{T} / \nu_{\mathrm{ref}}\right) \cdot x_{\mathrm{ref}}\right)}{\left(1-\left(\nu_{T} / \nu_{\mathrm{ref}}\right) \cdot x_{D, \mathrm{ref}}\right)}-X_{D, i}\right)+\widetilde{W} \cdot\left(X_{i} \cdot \frac{\left(1-\left(\nu_{T} / \nu_{\mathrm{ref}}\right) \cdot x_{\mathrm{ref}}\right)}{\left(1-\left(\nu_{T} / \nu_{\mathrm{ref}}\right) \cdot x_{W, \mathrm{ref}}\right)}-X_{W, i}\right)-\widetilde{F}_{d} \cdot \\
\left(X_{i} \cdot \frac{\left(1-\left(\nu_{T} / \nu_{\mathrm{ref}}\right) \cdot x_{\mathrm{ref}}\right)}{\left(1-\left(\nu_{T} / \nu_{\mathrm{ref}}\right) \cdot x_{f d, \mathrm{ref}}\right)}-X_{f d, i}\right)-\widetilde{F}_{w} \cdot\left(X_{i} \cdot \frac{\left(1-\left(\nu_{T} / \nu_{\mathrm{ref}}\right) \cdot x_{\mathrm{ref}}\right)}{\left(1-\left(\nu_{T} / \nu_{\mathrm{ref}}\right) \cdot x_{f w, \mathrm{ref}}\right)}-X_{f w, i}\right)-X_{i} \cdot\left(1-\left(\frac{\nu_{T}}{\nu_{\mathrm{ref}}}\right) \cdot x_{\mathrm{ref}}\right) \cdot \nu_{T} \cdot \\
\int_{\text {column }}\left(\frac{d \xi}{d t}\right) d h+U \cdot X_{i} \cdot\left(\frac{\nu_{T}}{\nu_{\text {ref }}}\right) \cdot\left(\frac{d x_{\mathrm{ref}}}{d t}\right)\end{array}$ \\
\hline
\end{tabular}

The following transformation, proposed by Doherty and Buzad [14], is used when computing a vessel composition path:

$$
\begin{gathered}
X_{i}=\frac{x_{i}-\left(\nu_{i} / \nu_{\mathrm{ref}}\right) \cdot x_{\mathrm{ref}}}{1-\left(\nu_{T} / \nu_{\mathrm{ref}}\right) \cdot x_{\mathrm{ref}}} \\
Y_{i}=\frac{y_{i}-\left(\nu_{i} / \nu_{\mathrm{ref}}\right) \cdot y_{\mathrm{ref}}}{1-\left(\nu_{T} / \nu_{\mathrm{ref}}\right) \cdot y_{\mathrm{ref}}}
\end{gathered}
$$

where $v_{i}$ is stoichiometric coefficient of component $i$ in the reaction, $\nu_{T}$ is the sum (total) of all the stoichiometric coefficients in the reaction, and the index ref refers to a reference component. Using this transformation together with the transformation shown in (1), proposed by Stéger et al. [2], the vessel path equation is brought to a form similar to that used for the nonreactive vessel by Lelkes et al. [10] and Varga et al. [12]. The equations have the same form if the total change of moles in the reaction is zero. This is shown in Table 2. Here $U$ denotes the number of moles in the vessel, $\xi$ denotes reaction coordinate, and the sum of the reaction coordinates is made along the column. Indices $D$ and $W$ refer to distillate and weight product, respectively; indices fd and fw refer to feed streams entering the rectifier part and the stripper part, respectively.
In order to analyze all the potential column sections, the section profiles and the still path are modeled with the equations presented in Table 1. A configuration is feasible if the specified products can be produced with a reasonable recovery at some operation parameter values (reflux ratio, entrainer feed ratio, etc.), that is, in a reasonable time while the vessel composition continuously changes. In each moment of this process, the column profile must connect the vessel with the specified product compositions. In order to maintain such a connection, the individual section profiles must meet at the section junctions, and the profiles in those sections neighboring the vessel must meet the vessel composition in some sense. This may not only mean simply an intersection of the profiles (and intersection of some profiles with the vessel path) but may also mean a more complicated criterion for reactive sections and reactive vessel path. The conditions of the feasibility of the junction points are presented in detail in Stéger et al. [2] and summarized briefly in Table 3.

If a nonreactive section is directly situated above a reactive section, then the dew-point profile of the upper section must intersect the liquid composition profile of the lower section. If a nonreactive section is directly situated above a reactive vessel, then the dew-point profile of this section must intersect the reactive vessel path. The dew point profile is 
TABLE 3: Conditions of feasibility at column section junctions [2].

Contact of two nonreactive sections

Contact of two reactive sections

A nonreactive section above a reactive section

A reactive section above a nonreactive section

Contact between a nonreactive section and a nonreactive vessel

Contact between a reactive section and a reactive vessel

Contact between a nonreactive stripping section and a reactive still

Contact between a nonreactive rectification section and a reactive vessel
Intersection of the compositions profiles

Intersection of the compositions profiles

Intersection of the dew-point profile of the upper section with the composition profile of the lower section

Intersection of the compositions profiles

Intersection of the composition profile and the vessel path Intersection of the composition profile and the vessel path

Intersection of the composition profile and the vessel path

Intersection of the dew-point profile and the reactive vessel path the curve of imaginary liquid compositions in equilibrium with the vapor compositions formed in the section. Note that the vapor composition profile is computed from the liquid composition profile by material balance, and; thus, the dew point profile is different from the liquid composition profile.

The feasibility criterion is the intersection of the liquid composition profiles or a profile and the vessel path in all the other cases.

2.2. Multiple Reaction Systems. The transformation of component flow rates is extended to multireaction systems, as

$$
\widetilde{\mathbf{a}}_{n}=\mathbf{a}_{n}-\mathbf{A} \cdot \mathbf{B}^{-1} \cdot \mathbf{a}_{n}^{(\mathrm{ref})}
$$

where index $n$ refers to stage $n$, matrix $\mathbf{A}$ is an $C \times R$ matrix of stoichiometric coefficients of the components in the reactions, $\mathbf{B}$ is an $R \times R$ matrix of stoichiometric coefficients of the reference components, $C$ is the number of components, $R$ is the number of reactions, $a_{n}$ is a component flow rate vector of all the $C$ components, whereas $\mathbf{a}_{n}^{(\text {ref })}$ is a component flow rate vector of the $R$ reference components:

$$
\mathbf{a}_{n} \equiv\left[\begin{array}{c}
a_{n, 1} \\
\vdots \\
a_{n, i} \\
\vdots \\
a_{n, N c}
\end{array}\right] \mathbf{a}_{n}^{(\mathrm{ref})} \equiv\left[\begin{array}{c}
a_{n, 1}^{(\mathrm{ref})} \\
\vdots \\
a_{n, i}^{(\mathrm{ref})} \\
\vdots \\
a_{n, R}^{(\mathrm{ref})}
\end{array}\right] .
$$

The column profile equations given in Table 1 remain unchanged, due to the transformation. follows.

The form of the reactive vessel path equation become as

$$
\text { If } \nu_{T}=0 \text {, then }
$$

$$
\begin{aligned}
\frac{d \mathbf{X}_{i}}{d t}= & D\left(\mathbf{X}_{i}-\mathbf{X}_{D, i}\right)+W\left(\mathbf{X}_{i}-\mathbf{X}_{W, i}\right) \\
& -F_{d}\left(\mathbf{X}_{i}-\mathbf{X}_{d f, i}\right)-F_{w}\left(\mathbf{X}_{i}-\mathbf{X}_{w f, i}\right),
\end{aligned}
$$

else $\left(\nu_{T} \neq 0\right)$

$$
\begin{aligned}
\tilde{U}= & U\left(1-v_{T}^{T} \mathbf{N}_{(\mathrm{ref})}^{-1} \mathbf{x}^{(\mathrm{ref})}\right) \\
\widetilde{U} \frac{d}{d t} \mathbf{X}_{i}= & \widetilde{D}\left(\mathbf{X}_{i} \frac{1-\mathbf{b}^{T} \mathbf{x}^{(\mathrm{ref})}}{1-\mathbf{b}^{T} \mathbf{x}_{D}^{(\mathrm{ref})}}-\mathbf{X}_{D, i}\right) \\
& +\widetilde{W}\left(\mathbf{X}_{i} \frac{1-\mathbf{b}^{T} \mathbf{x}^{(\mathrm{ref})}}{1-\mathbf{b}^{T} \mathbf{X}_{W}^{(\mathrm{ref})}}-\mathbf{X}_{W, i}\right) \\
& -\widetilde{F}_{d}\left(\mathbf{X}_{d f, i} \frac{1-\mathbf{b}^{T} \mathbf{x}^{(\mathrm{ref})}}{1-\mathbf{b}^{T} \mathbf{x}_{d f}^{(\mathrm{ref})}}-\mathbf{X}_{d f, i}\right) \\
& -\widetilde{F}_{w}\left(\mathbf{X}_{w f, i} \frac{1-\mathbf{b}^{T} \mathbf{x}^{(\mathrm{ref})}}{1-\mathbf{b}^{T} \mathbf{x}_{w f}^{(\mathrm{ref})}} \mathbf{X}_{w f, i}\right) \\
& -v_{T}^{T} \mathbf{X}_{i}\left(1-\mathbf{b}^{T} \mathbf{x}^{(\mathrm{ref})}\right) \int_{\text {column }} \frac{d \xi}{d t} d h \\
& +U \mathbf{X}_{i} \mathbf{b}^{T} \frac{d}{d t} \mathbf{x}^{(\mathrm{ref})},
\end{aligned}
$$

where $v_{T}^{T}$ is the transpose of vector $\nu_{T}$ of total stoichiometric coefficients, of length $R, \mathbf{N}_{(\text {ref })}$ is an $R \times R$ matrix of stoichiometric coefficients of the reference components in each reaction, and $\mathbf{b}^{T}$ is the transpose of vector $\mathbf{b}$ defined by

$$
\mathbf{b}^{T} \equiv \mathbf{B}^{-1} v_{T}^{T}
$$

The model equations and the vessel path of multireaction systems are derived in details in Appendices A and B, respectively.

\section{Feasibility Study}

Application of the equations and criteria presented in Section 1 is demonstrated here by performing the feasibility study of transesterification of dimethyl carbonate (DMC) with ethanol $(\mathrm{EtOH})$ in a batch extractive distillation process. 
Transesterification of DMC consists of two cascade reversible reactions with an intermediate methyl ethyl carbonate (MEC) [9]. The main product is diethyl carbonate
(DEC), and methanol (MeOH) is a by-product, but both of them are considered as desired products in our case as follows:
$\begin{gathered}\mathrm{H}_{3} \mathrm{COCOOCH}_{3} \\ \mathrm{DMC}\end{gathered}+\underset{\mathrm{EtOH}}{\mathrm{C}_{2} \mathrm{H}_{5} \mathrm{OH}} \stackrel{\mathrm{K}_{1}}{\Longleftrightarrow} \begin{gathered}\mathrm{H}_{3} \mathrm{COCOOC}_{2} \mathrm{H}_{5} \\ \mathrm{MEC}\end{gathered}+\begin{gathered}\mathrm{CH}_{3} \mathrm{OH} \\ \mathrm{MeOH}\end{gathered}$

$\underset{\mathrm{MEC}}{\mathrm{H}_{3} \mathrm{COCOOC}_{2} \mathrm{H}_{5}}+\underset{\mathrm{EtOH}}{\mathrm{C}_{2} \mathrm{H}_{5} \mathrm{OH}} \stackrel{\mathrm{K}_{2}}{\Longleftrightarrow} \quad \begin{gathered}\mathrm{H}_{5} \mathrm{C}_{2} \mathrm{OCOOC}_{2} \mathrm{H}_{5} \\ \text { DEC }\end{gathered}+\begin{gathered}\mathrm{CH}_{3} \mathrm{OH} \\ \mathrm{MeOH}\end{gathered}$.

The equilibrium-limited reactions are characterized with equilibrium constants $K_{1}=2.33$ and $K_{2}=0.26$ obtained by our laboratory measurements and are approximately constant in the studied temperature range. The equilibrium constants of the consecutive reactions are described by the following equations:

$$
\begin{aligned}
& K 1=\frac{[\mathrm{MEC}] \cdot[\mathrm{MeOH}]}{[\mathrm{DMC}] \cdot[\mathrm{EtOH}]}=2.33, \\
& K 2=\frac{[\mathrm{DEC}] \cdot[\mathrm{MeOH}]}{[\mathrm{MEC}] \cdot[\mathrm{EtOH}]}=0.26 .
\end{aligned}
$$

Total conversion cannot be reached because the reactions are equilibrium limited, but the productivity can be increased by removing the reaction products from the reaction space. The objective of this study is to examine the feasibility of simultaneously producing $\mathrm{MeOH}$ and DEC in middlevessel column. Both product purities are specified to 0.95 $\left(x_{d} \geq 0.95, x_{w} \geq 0.95\right)$.

Vapor-liquid equilibrium is modeled with the modified Raoult-Dalton equation

$$
y_{i} P=\gamma_{i} x_{i} p_{i}^{o}
$$

where $P$ is the actual pressure, $\gamma_{i}$ is activity coefficient of component $i$ in the liquid mixture, and $p_{i}^{o}$ is vapor pressure of pure component $i$. The activity coefficients are computed with 2-parameter Wilson model [15], and the vapor pressures with the Antoine equation in the form of

$$
\ln p_{i}^{o}=A-\frac{B}{T+C} .
$$

The model parameters are collected in Tables 4 and 5. The studied system contains 3 binary azeotropes, and there is no ternary azeotrope, as is shown in Table 6 [9].

Feasibility study aims at determining a region of vessel compositions, called "feasible region", from where the specified products can be reached by column profiles and the domain of column parameters such as reflux ratio $R$, reboil ratio $S$, and feed ratio(s) $F_{d} / V$ and/or $F_{w} / L$. The potential combinations of column sections appropriate to produce the desired products constitute the candidate column structures.
TABle 4: Parameters of the Wilson model (cal/mol) [7].

\begin{tabular}{lcc}
\hline & $\left(\lambda_{12}-\lambda_{11}\right)$ & $\left(\lambda_{22}-\lambda_{21}\right)$ \\
\hline MeOH-EtOH & 135.8113 & -132.0576 \\
MeOH-DMC & 771.98 & 202.44 \\
MeOH-MEC & 684.591 & 203.922 \\
MeOH-DEC & 364.177 & 411.055 \\
EtOH-DMC & 522.235 & 450.581 \\
EtOH-MEC & 709.523 & 164.462 \\
EtOH-DEC & 626.606 & 331.781 \\
DMC-MEC & 323.814 & -311.777 \\
DMC-DEC & -474.659 & 1000.0 \\
MEC-DEC & 80.9023 & -83.1232 \\
\hline
\end{tabular}

TABle 5: The Antoine parameters. $p_{i}^{o}$ is measured in $(\mathrm{mmHg})$, and $T$ is measured in [K] [7].

\begin{tabular}{lccc}
\hline & $A$ & $B$ & $C$ \\
\hline MEC & 16.82245 & 3376.596 & -49.461 \\
$\mathrm{MeOH}$ & 18.58752 & 3626.548 & -34.29 \\
$\mathrm{EtOH}$ & 18.91198 & 3800.417 & -41.67 \\
$\mathrm{DEC}$ & 15.56109 & 2817.835 & -84.304 \\
$\mathrm{DMC}$ & 16.82936 & 3253.553 & -44.25 \\
\hline
\end{tabular}

3.1. Total Reflux/Reboil Analysis. Running the system with no product removal is the limit case of any batch distillation process and is usually analyzed before finite reflux ratios and/ or finite reboil ratios are studied because total reflux and/or total reboil involves the highest reachable product purity in conventional distillation systems. It is not always so with extractive and reactive distillation, but performing a total reflux/reboil analysis is worth because valuable information can be obtained this way on the behavior of the studied system.

3.1.1. Nonreactive Sections. Nonreactive rectifying profiles and stripping profiles run along nonreactive residue curves in case of total reflux or total reboil, respectively. Thus, residue curves are analyzed. 
TABLE 6: VLE singularities in the system of $\mathrm{MeOH}$, EtOH, DMC, MEC, and DEC.

\begin{tabular}{lccc}
\hline System & $\begin{array}{c}\text { Composition } \\
\text { (mole fractions) }\end{array}$ & $\begin{array}{c}\text { Temperature } \\
\left({ }^{\circ} \mathrm{C}\right)\end{array}$ & $\begin{array}{c}\text { Type of } \\
\text { singularity* }\end{array}$ \\
\hline DMC-MeOH & $(0.123 ; 0.877)$ & 63.7 & $\mathrm{UN}$ \\
$\mathrm{MeOH}$ & 1 & 64.5 & $\mathrm{~S}$ \\
DMC-EtOH & $(0.31 ; 0.69)$ & 74.9 & $\mathrm{~S}$ \\
MEC-EtOH & $(0.05 ; 0.95)$ & 78.3 & $\mathrm{~S}$ \\
EtOH & 1 & 78.5 & $\mathrm{~S}$ \\
DMC & 1 & 90.0 & $\mathrm{~S}$ \\
MEC & 1 & 109.2 & $\mathrm{~S}$ \\
DEC & 1 & 126.0 & $\mathrm{SN}$ \\
\hline
\end{tabular}

${ }^{*}$ SN: stable node; UN: unstable node; S: saddle.

The studied system consists of five components. Thus, visualization of the residue curves is difficult. With the knowledge of the boiling points of the azeotropes and the pure components, the singular points and, thus, the shape of the residue curve map of the nonreactive system can be determined. In order to make the visualization easier, the system is projected to a plane (Figure 2). According to this projected network, six-saddle points, one stable-node (SN), and one unstable node (UN) are found.

The desired products are DEC as bottom product and $\mathrm{MeOH}$ as distillate. Since the DEC vertex is the stable node of the system, production of pure DEC in the bottom is feasible both in batch and continuous processes. Just the contrary, pure $\mathrm{MeOH}$ cannot be produced at the top of a nonreactive rectifying section because the $\mathrm{MeOH}$ vertex is a saddle point (S) of the system. The top product would be the unstable node, that is, the DMC-MeOH azeotrope.

Such a qualitative analysis of the nonreactive extractive profiles is rather difficult because their behavior cannot be visualized in a similar and clear way. Accumulation points (stable nodes) of the extractive profile bundles depend on process parameters like reflux ratio $R$ or reboil ratio $S$ and feed ratio $F / V$ or $F / L$.

3.1.2. Reactive Sections. Due to the instantaneous reaction equilibrium, dimensionality of the reactive material system is reduced to a $2 \mathrm{D}$ hypersurface (of the equilibrium points) in the originally $4 \mathrm{D}$ linear space of the mole fractions. (There are five mole fractions, their sum is unity, and; thus, only four independent mole fractions are considered.) This curved hypersurface is referred to as "the reactive surface". Due to applying the proposed transformation, this reactive surface, represented with reactive residue curves, is projected to a $2 \mathrm{D}$ plane as is shown in Figure 3.

The reactive space contains the same unstable node (the locus of the DMC-MeOH azeotrope) and the same stable node (DEC vertex) as in the case of the nonreactive system. Thus, without extractive section, the DMC-MeOH azeotrope and pure DEC are withdrawn as distillate and bottom products, respectively, with infinite number of theoretical stages.
There is a narrow bundle of reactive residue curves running so near the $\mathrm{MeOH}$ vertex that they intersect the composition region of assigned purity. Thus, the assigned purity can be reached with a finite number of rectifying stages. The feasible region is the set of compositions covered by that part of this bundle which connect vertex DEC with the targeted region around vertex $\mathrm{MeOH}$.

Unfortunately, the targeted $\mathrm{MeOH}$ purity can be reached just with a rather narrow bundle of profiles running along the DEC- EtOH edge and the EtOH-MeOH edge and cannot be reached from the central part of the space where DMCEtOH charge compositions are expected. Even with a finite number of rectifying stages, such a high EtOH concentration must be maintained in the vessel in order to keep the EtOH composition in the feasible region that makes this opportunity practically useless.

Such a deficiency is usually overcome by an extractive section, according to the lessons learned from experience in extractive distillation. If a stable node of the extractive profiles is situated nearer the DEC-EtOH edge or the EtOH$\mathrm{MeOH}$ edge than a candidate curve runs, then a wide bundle of extractive profiles started from the middle part of the reactive space will intersect that candidate curve. Thus, the targeted pure $\mathrm{MeOH}$ vertex can be approached from within a wide region of potential vessel compositions by a reactive extractive section and a reactive rectifying section above it.

$\mathrm{EtOH}$ is a good candidate to play the role of the entrainer because, according to experience collected in earlier feasibility studies, the stable node of the extractive profiles is shifted from the lightest point of the system toward the entrainer vertex with increasing feed ratio. Thus, one expects the stable node to approach either the EtOH-MeOH edge or the DEC$\mathrm{EtOH}$ edge with increasing the flow rate of the $\mathrm{EtOH}$ feed. Finally, EtOH would be a preferable entrainer because it is already a component of the reaction system, and; thus, no foreign component would be involved.

How the stable node is shifted with the feed ratio $F / V$ according to calculations (at total reflux) is shown in Figure 4. It is located at the DMC-MeOH azeotrope when no entrainer is fed, and is practically located in the EtOH$\mathrm{MeOH}$ edge at and above $F / V=0.3$. The bold line in the figure represents an arbitrarily selected candidate curve. As is shown in Figure 5, all the extractive profiles started from potential charge locations run to the stable node and, thus, intersect the candidate line, that is, the candidate rectifying/stripping profile, at $F / V=0.3$. Thus, with a feed ratio higher than 0.3 , the desired purity can be reached from the whole quadrangle, that is, from any initial composition of the charge including the stoichiometric composition.

It is also possible to withdraw DEC as bottom product in continuous process, but it is not possible to withdraw pure $\mathrm{MeOH}$ as distillate without entrainer feeding.

3.2. Finite Reflux Analysis. Due to the high dimensionality of the nonreactive system, the nonreactive column profiles are rather difficult to visualize and analyze. Therefore, only the fully reactive column configurations are analyzed here with finite reflux and finite reboil ratios. 

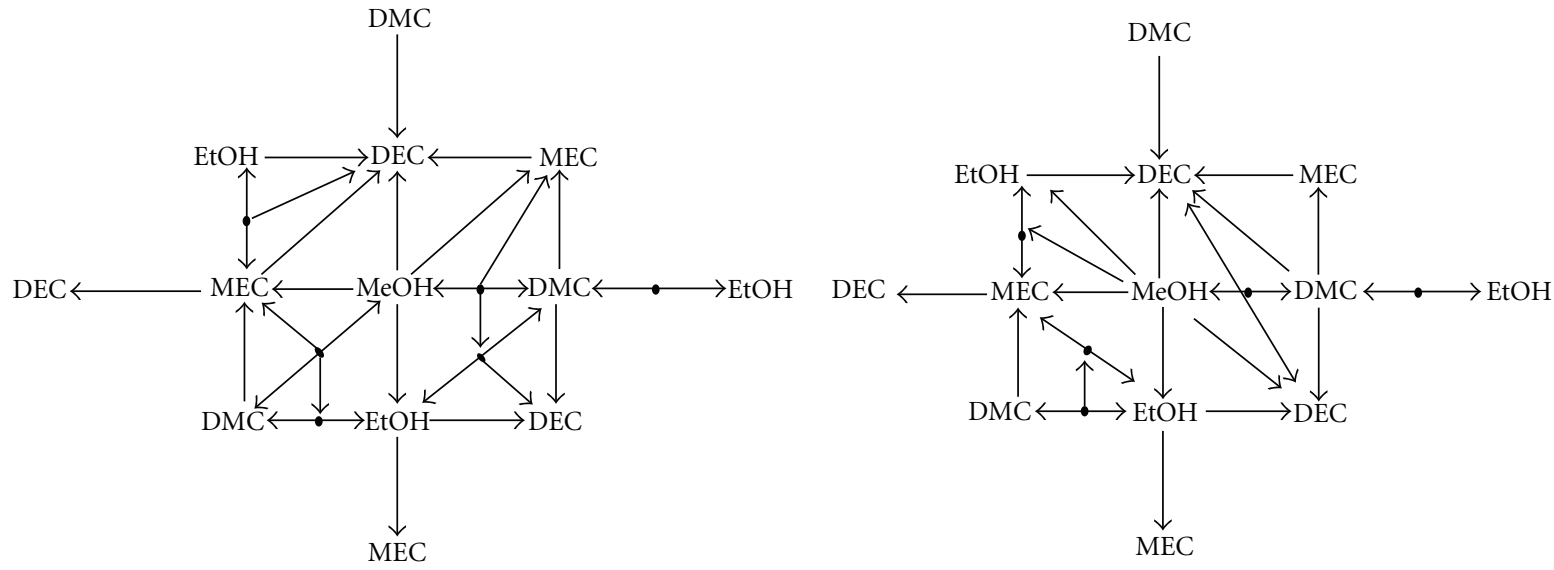

FIgure 2: The nonreactive system unfolded in 2D.

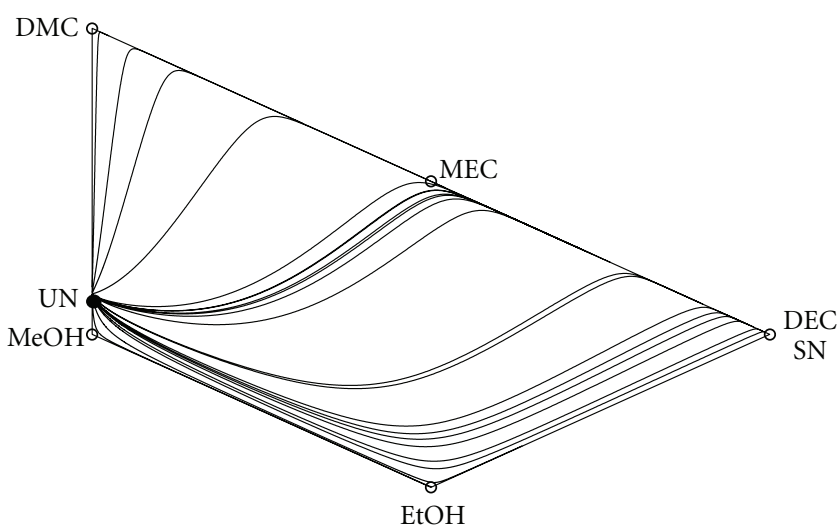

FIGURE 3: Residue curves in the transformed reactive space.

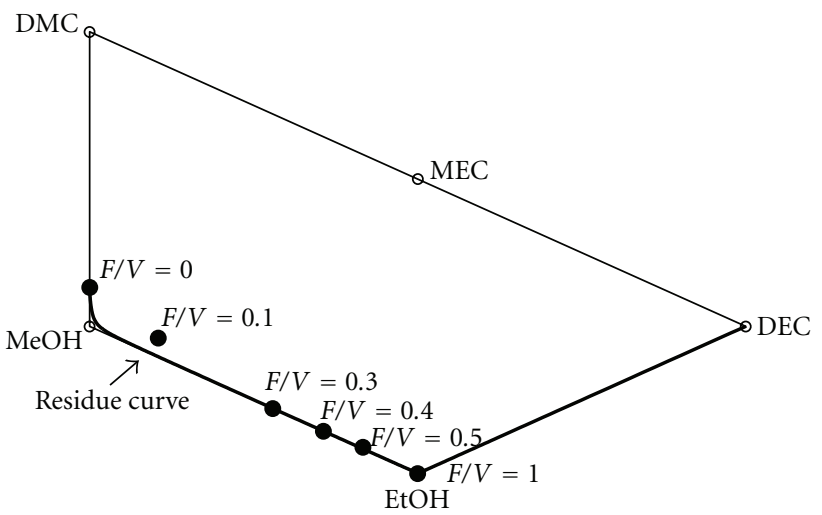

FIGURE 4: Shifting of the stable node of the extractive profiles at total reflux.

3.2.1. Effect of Reflux Ratio $R$ and Upper Feed Ratio $F_{d} / V$. Reactive rectifying profiles with finite reflux ratio are computed with the equations shown in Table 1. Numerical integration of the differential equation is started from an initial point inside the targeted purity region near the $\mathrm{MeOH}$ vertex. At $R \leq 4$, the profile runs along the EtOH-MeOH edge

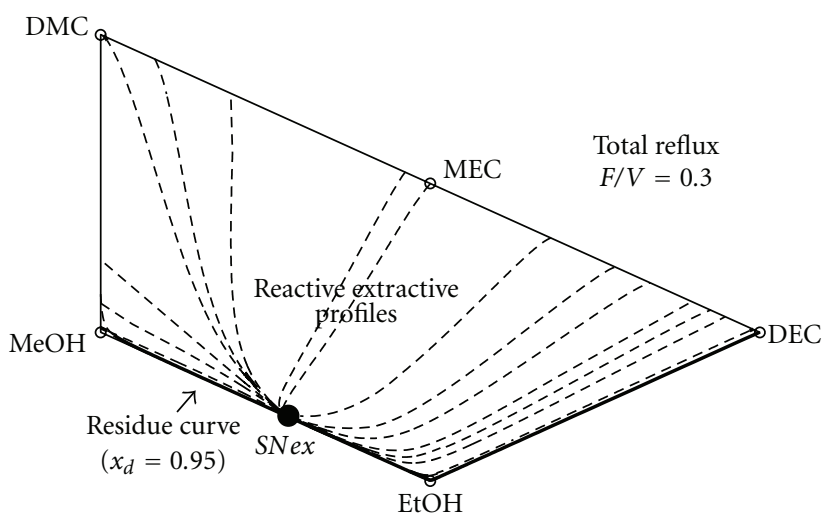

FIGURE 5: Reactive extractive profiles map at total reflux and feed ratio $F / V=0.3$.

but does not reach the EtOH vertex. At reflux ratios $R \geq 5$ the profiles are longer, turn toward the MEC-DEC edge, and run along it before reaching the accumulation point. $R=5$ can be considered as a minimum reflux ratio (Figure 6). The feasibility region without extractive section is, thus, a composition area delimited by the MEC-DEC, DEC-EtOH, and $\mathrm{EtOH}-\mathrm{MeOH}$ edges and the rectifying profile computed with $R=5$. Producing pure $\mathrm{MeOH}$ as distillate without extractive section is in principle possible at $R \geq 5$ from a narrow interval of the middle-vessel composition.

However, maintaining feasible region's compositions in the vessel would require a large concentration and amount of $\mathrm{EtOH}$, and this would give rise to a huge-volume vessel. Economy of the process is expected to improve by applying EtOH entrainer and an extractive section, according to the results of the total reflux analysis in Section 2.1 .

Reactive extractive profiles with finite reflux ratio and feed ratio are computed with the equations shown in Table 1. The initial compositions are selected near an edge of the reaction quadrangle. A few computed extractive profiles together with the rectifying profile are shown in Figure 6 at $R=5$ and $F_{d} / V=0.2$. 


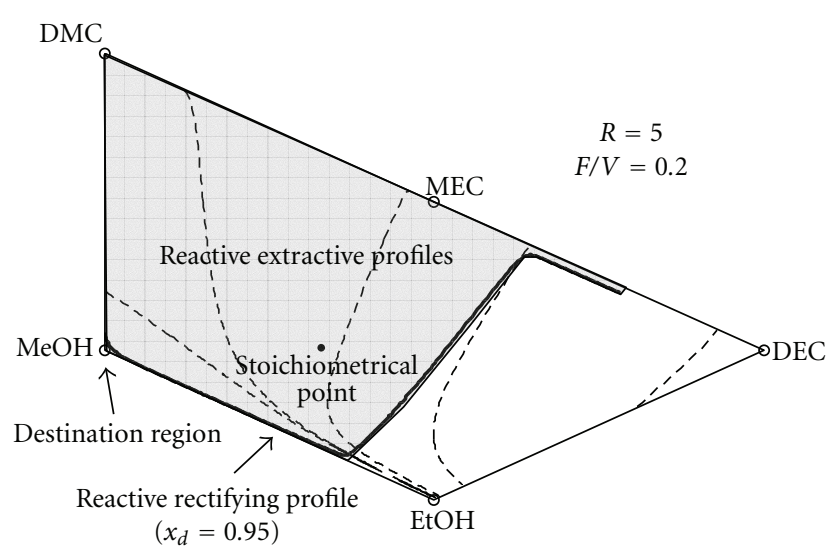

FIGURE 6: Rectifying profiles with finite reflux ratios.

At feed ratio $F_{d} / V=0.2$, all the extractive profiles run toward a point outside the quadrangle, and all the extractive profiles initiated from outside the feasible region of the rectifying profiles intersect the actual rectifying profile. When the vessel composition is situated toward the DMC$\mathrm{MeOH}$ edge from the reactive rectifying profile including the stoichiometric charge composition, the products can be reached with a combination of an extractive section and a rectifying section. Thus, a wide feasible region, shown with shading in the figure, is found.

\subsubsection{Effect of Stripping Ratio $S$ and Lower Feed Ratio $F_{w} / L$.} Reactive stripping profiles with finite reboil ratio are computed with the equations shown in Table 1. Numerical integration of the differential equation is started from an initial point inside the targeted purity region near the DEC vertex. As the stripping ratio is decreased, the profiles become shorter. The stripping profile shown in Figure 7 is computed to the limit-purity specification; the profiles belonging to purer DEC run between the shown profiles and the DEC-EtOH edge and then between the final sections of the shown profile and the $\mathrm{MeOH}-\mathrm{EtOH}$ edge. This is a rather narrow feasible region, which does not connect the potential stoichiometric vessel composition and the destination region.

Applying an extractive section with EtOH as entrainer may extend the feasible region even in this case. Reactive extractive profiles with finite reboil ratio and feed ratio are computed with the equations shown in Table 1. The initial compositions are selected near an edge of the reaction quadrangle. A few computed extractive profiles together with the stripping profile are shown in Figure 7 at $S=10$ and $F_{w} / L=$ 0.3 .

With feed ratio $F_{w} / L=0.3$, the extractive profiles (drawn by dashed lines) run toward two different attractors somewhere outside the quadrangle and leave the reaction space through the DEC-EtOH edge near vertex DEC and through the $\mathrm{MeOH}-\mathrm{EtOH}$ edge near vertex EtOH. Most of the extractive profiles intersect the stripping profile and connect the feasible region with a large interval of potential vessel compositions, including the stoichiometric one.

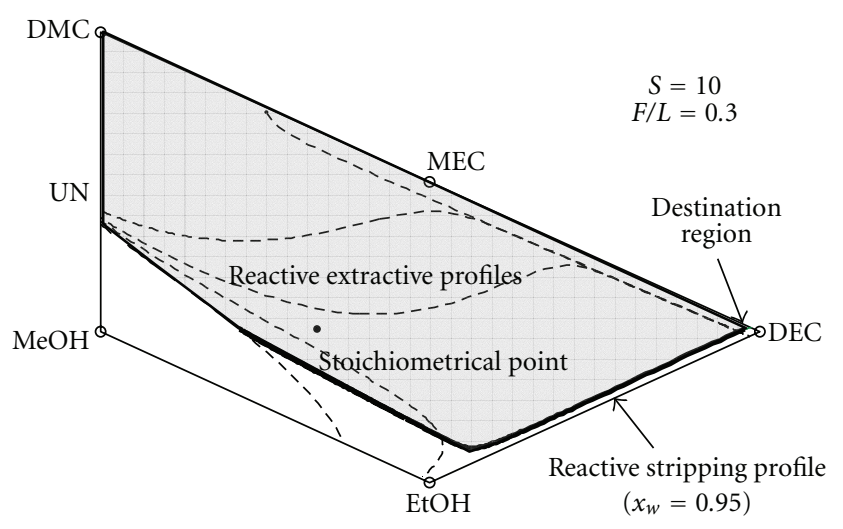

Figure 7: Reactive extractive profiles at $S=10$ and at $F_{w} / L=0.3$.

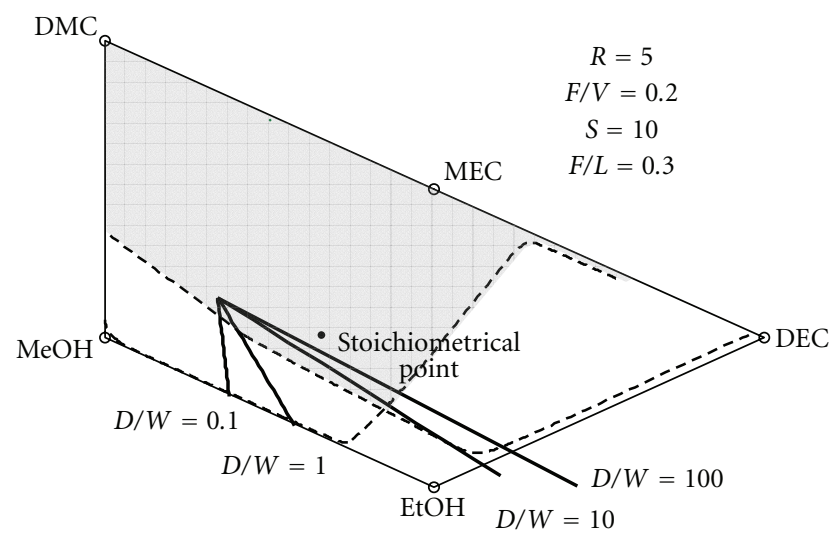

FIGURE 8: Feasible region of middle-vessel column with two extractive sections.

3.2.3. Effect of Production Ratio $D / W$ to the Vessel Path. The feasible region in a middle-vessel column is the region of feasible compositions in the middle vessel. These compositions should be feasible both for the upper part and the lower part; thus, the feasible region is the intersection of the two feasible regions determined for them. Considering extractive sections both above and below the vessel, the feasible region at $R=$ $5, S=10, F_{d} / V=0.2$, and $F_{w} / L=0.3$ is shown by shading in Figure 8.

The vessel path is the path of the evolving vessel composition during the process. The reactive still path is computed by integrating the equation shown in Table 2 from an arbitrarily selected initial point $x_{0}(\mathrm{DEC}, \mathrm{DMC}, \mathrm{EtOH}, \mathrm{MEC}, \mathrm{MeOH})=$ $(0.013,0.160,0.184,0.134,0.509)$. The vessel path runs in different directions according to varied product ratio $\mathrm{D} / \mathrm{W}$, as is shown in Figure 8. The vessel composition gets farthest from the DMC vertex while kept in the feasible region at about $D / W=10$.

If the initial point contains DMC and EtOH only, then the vessel composition turns toward the $\mathrm{MeOH}-\mathrm{EtOH}$ edge at middle product ratios and can be kept in the feasible region with very low product ratio only. Thus, it is easier to let it reach the border of the feasible region, parallel to the $\mathrm{MeOH}-\mathrm{EtOH}$ edge, and then apply the $D / W=10$ value. 
Table 7: Parameters of the simulations with ProSimBatch.

\begin{tabular}{|c|c|c|c|c|c|}
\hline Simulation \# & 1 & 2 & 3 & 4 & 5 \\
\hline$R$ & $\infty$ & $\infty$ & 5 & $\infty$ & 5 \\
\hline$S$ & $\infty$ & $\infty$ & $\infty$ & 10 & 10 \\
\hline$F_{d} / V$ & 0 & 0.3 & 0.2 & 0 & 0.2 \\
\hline$F_{w} / L$ & 0 & 0 & 0 & 0.3 & 0.3 \\
\hline$N_{\text {rect }}$ & 30 & 30 & 30 & 30 & 30 \\
\hline$N_{\text {upper ext }}$ & 16 & 16 & 16 & 16 & 16 \\
\hline$N_{\text {strip }}$ & 10 & 10 & 10 & 10 & 10 \\
\hline$N_{\text {lower ext }}$ & 10 & 10 & 10 & 10 & 10 \\
\hline$Q_{\text {cond }}$ & $4880 \mathrm{~W}$ & $4880 \mathrm{~W}$ & $4880 \mathrm{~W}$ & $1035 \mathrm{~W}$ & $1035 \mathrm{~W}$ \\
\hline$Q_{\text {reboil }}$ & $1020 \mathrm{~W}$ & $1020 \mathrm{~W}$ & $1020 \mathrm{~W}$ & $5180 \mathrm{~W}$ & $5180 \mathrm{~W}$ \\
\hline Hold-up (tray) & $0.35 \mathrm{~mol}$ & $0.35 \mathrm{~mol}$ & $0.35 \mathrm{~mol}$ & $30 \mathrm{~cm}^{3}$ & $30 \mathrm{~cm}^{3}$ \\
\hline Hold-up (MV) & $350 \mathrm{~mol}$ & $350 \mathrm{~mol}$ & $350 \mathrm{~mol}$ & $3000 \mathrm{~cm}^{3}$ & $3000 \mathrm{~cm}^{3}$ \\
\hline Charge & $4000 \mathrm{~mol}$ & $4000 \mathrm{~mol}$ & $4000 \mathrm{~mol}$ & $5125 \mathrm{~cm}^{3}$ & $5125 \mathrm{~cm}^{3}$ \\
\hline
\end{tabular}

\section{Validation by Rigorous Simulation}

The results of the feasibility study are validated by rigorous simulations performed using ProSimBatch, a dynamic simulator for batch distillation and reactive processes. This software product works by numerically integrating differential equations, and; thus, it can also compute the start-up phase of the process. Unfortunately, the method of the start-up procedure is fixed in the software, and is not a selectable option.

This simulator allows performing simulation of batchreactive distillation processes in a rectifier, in a stripper, or in a middle-vessel column. In the case of a middle-vessel column, an additional withdrawal has to be defined at the bottom of the column. The middle vessel is defined as a tray with large holdup; its leaving liquid flow rate and its heat duty can be specified as well.

Instantaneously equilibrium-limited reaction cannot be specified during the simulations. Instead, four kinetically controlled reactions are specified. The reaction rate coefficients are set large (to achieve rather fast reactions) in such a way that their ratios are equal to the equilibrium constants of the equilibrium limited reactions:

$$
K_{1}=\frac{k_{+1}}{k_{-1}}=2.33, \quad K_{2}=\frac{k_{+2}}{k_{-2}}=0.26 .
$$

For simulation, stage numbers should also have to be specified. In order to obtain initial estimates for the needed number of stages, preliminary calculations are performed. For this aim, not the simulator but a simple stage-by-stage calculating device is used. The calculations are started from the vessel composition, performed along the supposed extractive profile, and, when a composition similar to that obtained by the feasibility study is achieved, then the calculation is continued with the supposed rectifier or stripping profile until the specified purity of the product is reached. Considering the initial and final vessel compositions, the calculated minimum and maximum stage numbers are 30 and 56 in the rectifier section, 20 and 20 in the stripper section, 6 and 16 in the upper extractive section, and 5 and 10 in the lower extractive section.

4.1. Validation of the Reactive Profiles. Five column configurations are considered with different reflux and reboil ratios and with different feed ratios in order to compare the results of the simulated and the calculated profiles. The parameters applied in the simulations are collected in Table 7.

The simulated composition profiles along the column height are represented by thick lines in Figures 9 to 13. Some profile curves calculated in the feasibility study are represented by thin lines in these figures in order to compare the simulation results with these curve bundles. Middle-vessel compositions are labeled by letters MV.

Two cases are simulated with total reflux and reboil, no feed, and two arbitrarily selected vessel compositions, as shown in Figure 9. The dotted and the continuous thick lines represent the sections below and above the middle vessel, respectively. The residue curves calculated during the feasibility study (drawn by thin continuous lines) are also shown in the figure. The simulated reactive profiles are well approximated by the calculated curves.

Two composition profiles simulated with total reflux and reboil, and with an average feed ratio $\left(F_{d} / V=0.3\right)$ in the upper part of the middle vessel column, and with two arbitrarily selected reboiler compositions, are shown in Figure 10. The dotted, the dashed, and the continuous lines correspond, respectively, to the section below the middle vessel and the sections below and above the feed in the upper part of the middle vessel column. The (dashed) extractive profiles approach the stable node of the extractive profile curves' bundle, as is also shown by two thin dotted lines calculated during the feasibility study. The thick continuous lines, representing the simulated reactive rectifying profiles, run almost together along the $\mathrm{MeOH}-\mathrm{EtOH}$ edge and the EtOH-DMC edge (see also Section 3.1.2). According to this Figure, the results of the feasibility study provide a good estimation for the composition profile along the column. 


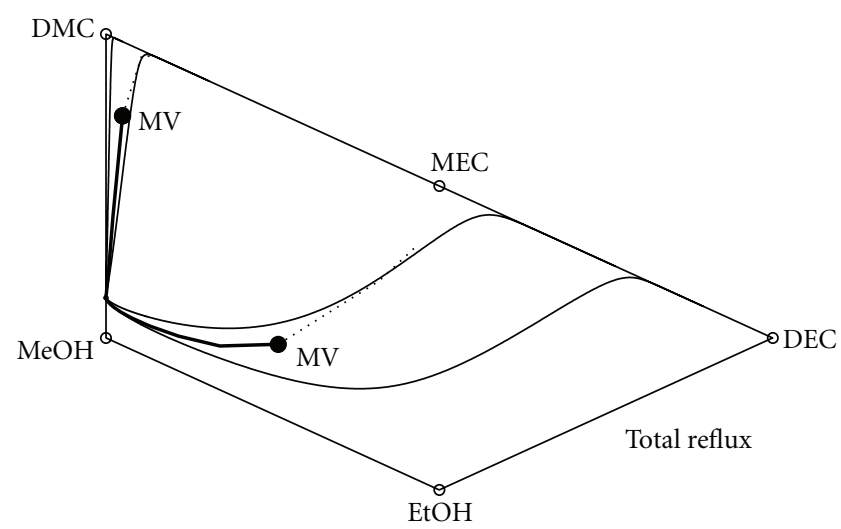

Sim. stripping pr.

- Sim. rect. pr.

— Cal. residue curve

FIgURE 9: Simulated and calculated residue curves.

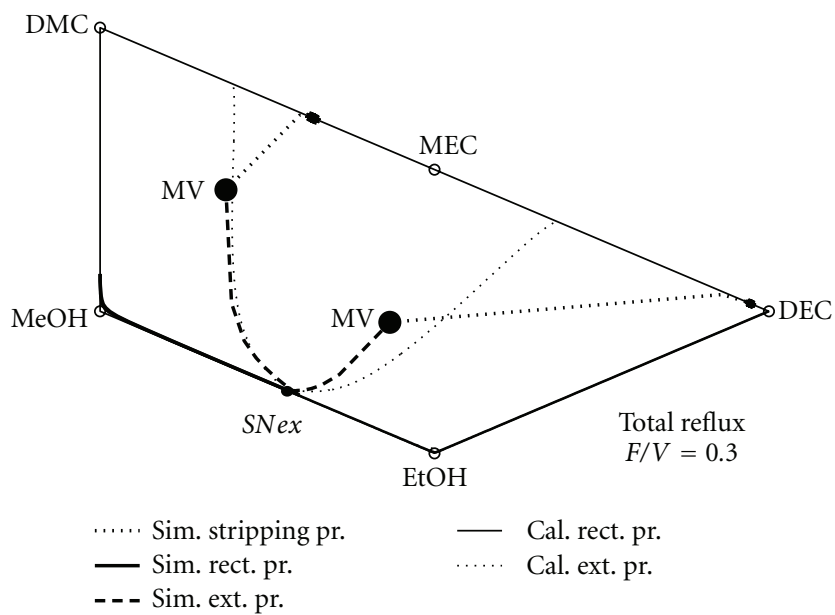

FIgURE 10: Simulated and calculated profiles at total reflux and reboil ratio, and at $F_{d} / V=0.3$.

A simulated column profile with total reboil, finite reflux ratio $(R=5)$, and with an average feed ratio $\left(F_{d} / V=0.2\right)$ is shown in Figure 11. The dotted, dashed, and the continuous thick lines correspond, respectively, to the stripping section below the middle vessel, and the sections below and above the feed in the upper part of the middle vessel column. The continuous and the dotted thin lines represent the reactive rectifying profile curve and a reactive upper extractive curve, respectively, as calculated in the feasibility study. The thin lines again provide good estimation to the simulated ones. The simulated rectifying and extractive profiles meet in a point nearer the $\mathrm{EtOH}$ vertex than anticipated because the simulated distillate composition is a little bit different from that specified in the feasibility calculations.

Two simulated column profiles with total reflux, finite reboil ratio $(S=10)$ and with an average feed ratio $\left(F_{w} / L=0.3\right)$ are shown in Figure 12. The dotted, the dashed, and the continuous thick lines, respectively, represent the rectifying section and the sections above and below the feed in the
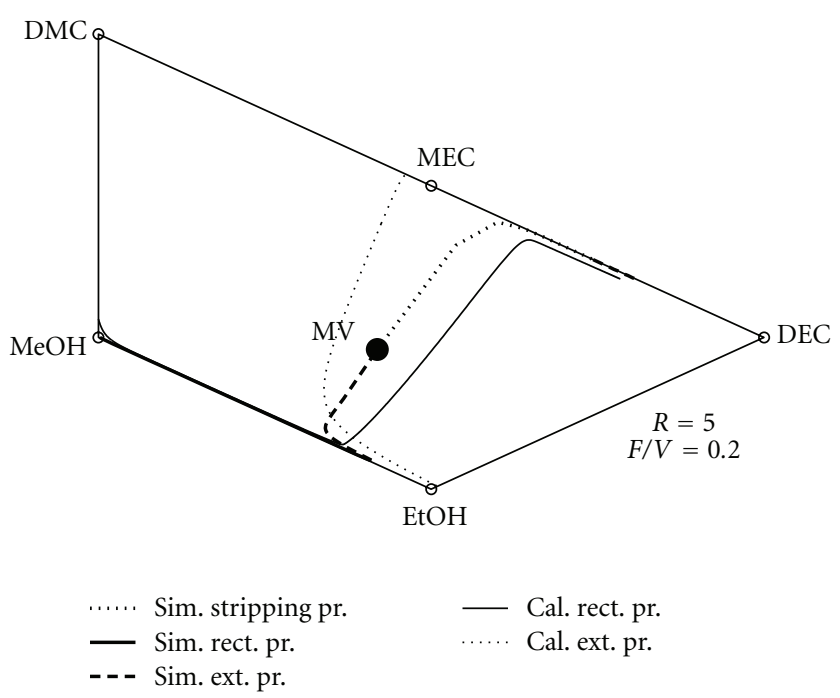

FIGURE 11: Simulated and calculated profiles at total reboil ratio, at $R=5$ and at $F_{d} / V=0.2$.

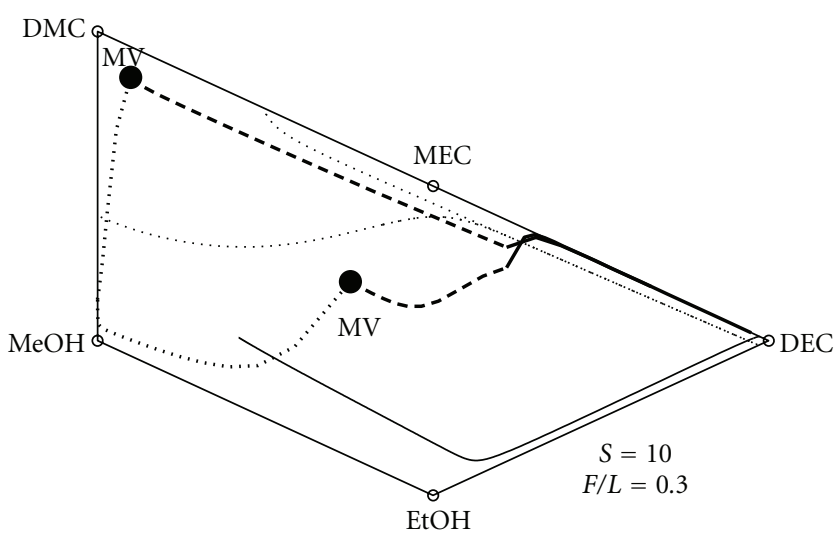

FIGURE 12: Simulated and calculated profiles at total reflux, at $S=$ 10 , and at $F_{w} / L=0.3$.

lower part of the middle vessel column. Two reactive stripping profile curves calculated in the feasibility study are shown by thin continuous lines, and two lower reactive extractive profile curves are also shown by thin dotted lines in this figure. The tendency of the calculated profile curves and the simulated profiles agree in this case, too.

Two simulated column profiles with finite reflux ratio $(R=5)$, finite reboil ratio $(S=10)$, upper feed ratio $F_{d} / V=0.2$, and lower feed ratio $\left(F_{w} / L=0.3\right)$ are shown in Figure 13. The simulated rectifying and stripping profiles and the simulated extractive profiles in the upper part and in the lower part are represented by thick continuous, dotted, and dashed lines, respectively. Profile curves calculated in the feasibility study are shown with thin lines. The calculated 


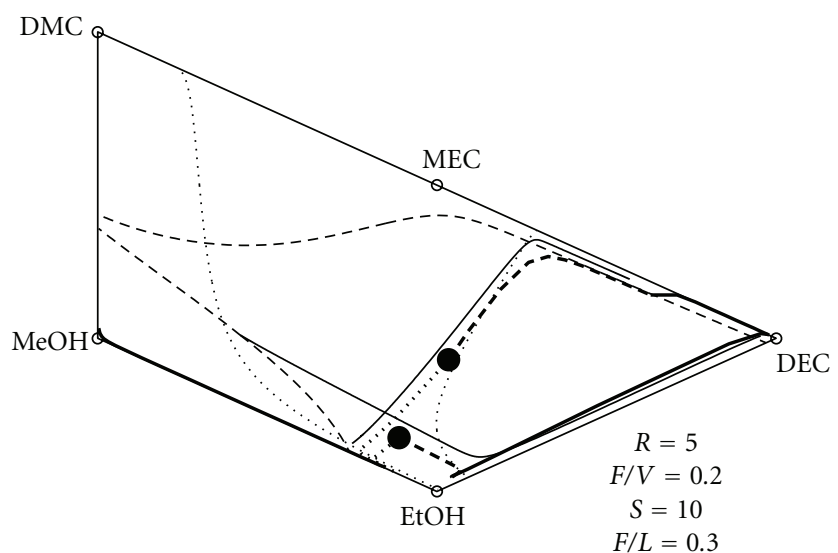
- Sim. rect. and stripping pr.
- - Sim. lower ext. pr.
.... Sim. upper ext. pr.
- Cal. rect. and stripping pr.
- - - Cal. lower ext. pr.
.... Cal. upper ext. pr.

FIgure 13: Simulated and calculated profiles during the feasibility study at $R=5$, at $F_{d} / V=0.2$, at $S=10$ and at $F_{w} / L=0.3$.

profile curves well approximate the simulated profiles in the lower part of the column but show deviation in the upper part because the simulated distillate composition is a little bit different from that specified in the feasibility calculations. However, the calculated profile curves give valid information on the qualitative shape of the simulated profiles even in this case.

\section{Conclusion}

The feasibility method of batch reactive distillation published in Stéger et al. [2] is extended for dealing with consecutive reactions. General model equations are derived for multireaction system with any number of chemical equilibriumlimited reactions. Since the presented general methodology is of graphical nature, performing the feasibility study of systems with more than 3 degrees of freedom might be difficult in practice. Thus, further investigation is needed for such, high complexity, systems.

The present methodology, applying some modifications, might be used to continuous processes, as well.

The presented methodology is demonstrated with the detailed study of the transesterification of dimethyl carbonate. Since the system contains 5 components, and the degree of freedom of any nonreactive section is 4 ; only the total reflux analysis is performed for the nonreactive sections. The feasibility analysis of the reactive sections is successfully performed. The proposed new transformation is used to present the graphical methodology applicable to multireaction and multicomponent system.

According to the presented feasibility study, at least one of the fully reactive middle-vessel column configurations is feasible. Extractive stages are needed both in the upper and lower parts of the column, with the following limiting parameters: $R \geq 5, F_{d} / V \geq 0.2, S \geq 10, F_{w} / L \geq 0.3$. However, only the configurations proposed by Stéger et al. [2]

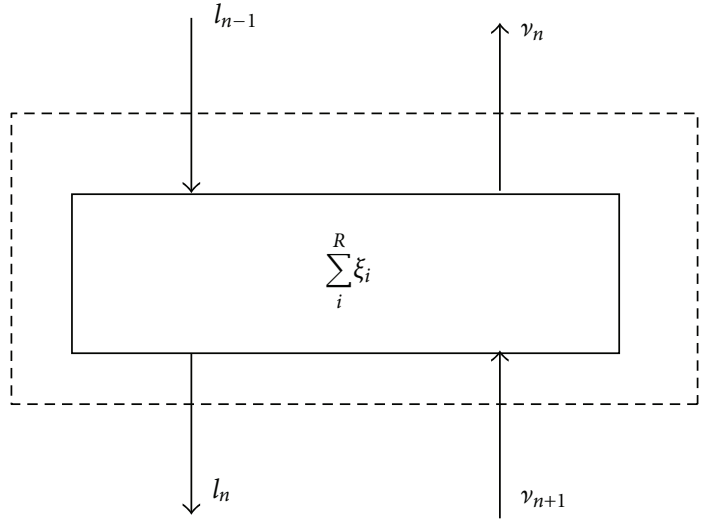

FIgURE 14: Theoretical tray.

have been investigated in the present work, and there may be other feasible configurations depending on the place and number of the applied feeds and reactive sections. Any of them can be studied with the presented method.

Effect of production ratio $(D / W)$ to the vessel path and feasible region of reactive vessel is also examined.

The results of the feasibility study are successfully validated by rigorous simulations.

\section{Appendices}

\section{A. Appendix A}

The component balance of any theoretical reactive tray with any number of reactions can be written as follows applying the reaction coordinates for the reactive term (see Figure 14)

$$
\begin{aligned}
& {\left[\begin{array}{c}
\vdots \\
v_{n, i} \\
\vdots
\end{array}\right]_{N}+\left[\begin{array}{c}
\vdots \\
l_{n, i} \\
\vdots
\end{array}\right]_{N}=\left[\begin{array}{c}
\vdots \\
v_{n+1, i} \\
\vdots
\end{array}\right]_{N}+\left[\begin{array}{c}
\vdots \\
l_{n-1, i} \\
\vdots
\end{array}\right]_{N}} \\
& +\left[\begin{array}{ccc}
\frac{\nu_{1, R 1}}{\nu_{\mathrm{ref} 1, R 1}} & \frac{\nu_{1, R 2}}{\nu_{\mathrm{ref} 2, R 2}} & \cdots \\
\frac{\nu_{2, R 1}}{\nu_{\mathrm{ref} 1, R 1}} & \frac{\nu_{2, R 2}}{\nu_{\mathrm{ref} 2, R 2}} & \cdots \\
\vdots & \vdots & \vdots
\end{array}\right]_{N \times R}\left[\begin{array}{c}
\vdots \\
\xi_{n, i} \\
\vdots
\end{array}\right]_{R} .
\end{aligned}
$$

In order to eliminate the reaction term, many reference components must be chosen as many reactions exist in the studied system $\left(N_{\text {ref }}=R\right)$. The reaction term can then be 
expressed using the component balance of the reference components as follows:

$$
\begin{aligned}
& {\left[\begin{array}{c}
\vdots \\
v_{n+1, i} \\
\vdots
\end{array}\right]_{N \text { ref }}+\left[\begin{array}{c}
\vdots \\
l_{n-1, i} \\
\vdots
\end{array}\right]_{N \text { ref }}}
\end{aligned}
$$

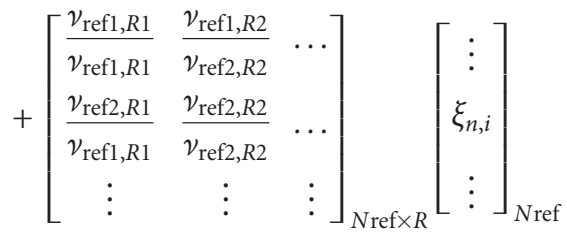$$
=\left[\begin{array}{c}
\vdots \\
v_{n, i} \\
\vdots
\end{array}\right]_{N \text { ref }}+\left[\begin{array}{c}
\vdots \\
l_{n, i} \\
\vdots
\end{array}\right]_{N \text { ref }}
$$$$
\mathbf{B}_{N \text { ref } \times R}^{-1}\left(\left[\begin{array}{c}
\vdots \\
v_{n+1, \text { ref }} \\
\vdots
\end{array}\right]_{N \text { ref }}+\left[\begin{array}{c}
\vdots \\
l_{n-1, \text { ref }} \\
\vdots
\end{array}\right]_{N \text { ref }}\right)
$$$$
+\left[\begin{array}{c}
\vdots \\
\xi_{n, \text { ref }} \\
\vdots
\end{array}\right]_{N \text { ref }}
$$$$
=\mathbf{B}_{N \text { ref } \times R}^{-1}\left(\left[\begin{array}{c}
\vdots \\
v_{n, \text { ref }} \\
\vdots
\end{array}\right]_{N \text { ref }}+\left[\begin{array}{c}
\vdots \\
l_{n, \text { ref }} \\
\vdots
\end{array}\right]_{N \text { ref }}\right) \text {, }
$$

$\left[\begin{array}{c}\vdots \\ \xi_{n, \text { ref }} \\ \vdots\end{array}\right]_{N \text { ref }}$

$$
\begin{gathered}
=\mathbf{B}_{N \text { ref } \times R}^{-1}\left(\left[\begin{array}{c}
\vdots \\
v_{n, \text { ref }} \\
\vdots
\end{array}\right]_{N \text { ref }}-\left[\begin{array}{c}
\vdots \\
v_{n+1, \text { ref }} \\
\vdots
\end{array}\right]_{N \text { ref }}\right. \\
\left.+\left[\begin{array}{c}
\vdots \\
l_{n, \text { ref }} \\
\vdots
\end{array}\right]_{N \text { ref }}+\left[\begin{array}{c}
\vdots \\
l_{n-1, \text { ref }} \\
\vdots
\end{array}\right]_{N \text { ref }}\right),
\end{gathered}
$$

where

$$
\begin{gathered}
\mathbf{B}=\left[\begin{array}{ccc}
\frac{\nu_{\mathrm{ref} 1, R 1}}{\nu_{\mathrm{ref} 1, R 1}} & \frac{\nu_{\mathrm{ref} 1, R 2}}{\nu_{\mathrm{ref} 2, R 2}} & \cdots \\
\frac{\nu_{\mathrm{ref} 2, R 1}}{\nu_{\mathrm{ref} 1, R 1}} & \frac{\nu_{\mathrm{ref} 2, R 2}}{\nu_{\mathrm{ref} 2, R 2}} & \cdots \\
\vdots & \vdots & \vdots
\end{array}\right]_{N \mathrm{ref} \times R}, \\
\mathbf{B}^{-1}=-\frac{\operatorname{Adj}(\mathbf{B})}{\operatorname{Det}(\mathbf{B})}
\end{gathered}
$$

The component balance now can be written by substituting the reaction term:

$$
\begin{aligned}
& {\left[\begin{array}{c}
\vdots \\
v_{n, i} \\
\vdots
\end{array}\right]_{N}+\left[\begin{array}{c}
\vdots \\
l_{n, i} \\
\vdots
\end{array}\right]_{N}} \\
& =\left[\begin{array}{c}
\vdots \\
v_{n+1, i} \\
\vdots
\end{array}\right]_{N}+\left[\begin{array}{c}
\vdots \\
l_{n-1, i} \\
\vdots
\end{array}\right]_{N} \\
& +\mathbf{A}_{N \times R} \mathbf{B}_{N \text { ref } \times R}^{-1} \\
& \times\left(\left[\begin{array}{c}
\vdots \\
v_{n, \text { ref }} \\
\vdots
\end{array}\right]_{N \text { ref }}-\left[\begin{array}{c}
\vdots \\
v_{n+1, \text { ref }} \\
\vdots
\end{array}\right]_{N \text { ref }}\right. \\
& \left.+\left[\begin{array}{c}
\vdots \\
l_{n, \mathrm{ref}} \\
\vdots
\end{array}\right]_{N \mathrm{ref}}+\left[\begin{array}{c}
\vdots \\
l_{n-1, \mathrm{ref}} \\
\vdots
\end{array}\right]_{N \mathrm{ref}}\right), \\
& {\left[\begin{array}{c}
\vdots \\
v_{n, i} \\
\vdots
\end{array}\right]_{N}-\mathbf{A}_{N \times R} \mathbf{B}_{N \text { ref } \times R}^{-1}\left[\begin{array}{c}
\vdots \\
v_{n, \text { ref }} \\
\vdots
\end{array}\right]_{N \text { ref }}} \\
& +\left[\begin{array}{c}
\vdots \\
l_{n, i} \\
\vdots
\end{array}\right]_{N}-\mathbf{A}_{N \times R} \mathbf{B}_{N \mathrm{ref} \times R}^{-1}\left[\begin{array}{c}
\vdots \\
l_{n, \mathrm{ref}} \\
\vdots
\end{array}\right]_{N \text { ref }} \\
& =\left[\begin{array}{c}
\vdots \\
v_{n+1, i} \\
\vdots
\end{array}\right]_{N}-\mathbf{A}_{N \times R} \mathbf{B}_{N \text { ref } \times R}^{-1}\left[\begin{array}{c}
\vdots \\
v_{n+1, \text { ref }} \\
\vdots
\end{array}\right]_{N \text { ref }}
\end{aligned}
$$




$$
\begin{gathered}
+\left[\begin{array}{c}
\vdots \\
l_{n-1, i} \\
\vdots
\end{array}\right]_{N} \\
-\mathbf{A}_{N \times R} \mathbf{B}_{N \text { ref } \times R}^{-1}\left[\begin{array}{c}
\vdots \\
l_{n-1, \text { ref }} \\
\vdots
\end{array}\right]_{N \text { ref }},
\end{gathered}
$$

where

$$
A=\left[\begin{array}{cc}
\frac{v_{1, R 1}}{v_{\text {refl } 1, R 1}} & \frac{v_{1, R 2}}{v_{\text {ref } 2, R 2}} \\
\frac{v_{2, R 1}}{v_{\text {ref1 } 1, R 1}} & \frac{v_{2, R 2}}{v_{\text {ref } 2, R 2}} \\
\vdots & \vdots
\end{array}\right]_{N \times R}
$$

Since the terms in the brackets are analogues, the following definitions of transformation can be introduced in order to simplify the relationship:

$$
\begin{gathered}
\tilde{v}_{n} \equiv\left[\begin{array}{c}
\vdots \\
\tilde{v}_{n} \\
\vdots
\end{array}\right]_{N}=\left[\begin{array}{c}
\vdots \\
v_{n, i} \\
\vdots
\end{array}\right]_{N}-\mathbf{A}_{N \times R} \mathbf{B}_{N \mathrm{ref} \times R}^{-1}\left[\begin{array}{c}
\vdots \\
v_{n, \mathrm{ref}} \\
\vdots
\end{array}\right]_{N \text { ref }}, \\
\tilde{\mathbf{l}}_{n} \equiv\left[\begin{array}{c}
\vdots \\
\tilde{l}_{n} \\
\vdots
\end{array}\right]_{N}=\left[\begin{array}{c}
\vdots \\
l_{n, i} \\
\vdots
\end{array}\right]_{N}-\mathbf{A}_{N \times R} \mathbf{B}_{N \mathrm{ref} \times R}^{-1}\left[\begin{array}{c}
\vdots \\
l_{n, \text { ref }} \\
\vdots
\end{array}\right]_{N \text { ref }} .
\end{gathered}
$$

The component balance can be written with these transformed variables as follows:

$$
\tilde{l}_{n}-\tilde{l}_{n-1}=\tilde{\nu}_{n+1}-\tilde{\nu}_{n} .
$$

The component flow rate can be approximated with its Taylor series written up to the first degree thus the differential of the component flow rate can be expressed (A.9). As the transformations presented by (A.7) are linear, the differential remains the same with the transformed variables (A.10) as follows:

$$
\begin{gathered}
\tilde{l}_{n}=\tilde{l}_{n-1}+\left.\frac{d \tilde{l}}{d h}\right|_{n-1} \cdot(n-(n-1)), \\
\frac{d \tilde{l}}{d h}=\tilde{l}_{n}-\tilde{l}_{n-1} .
\end{gathered}
$$

Using (A.8), the reactive profile in the column, expressing the driving force of the system, is obtained:

$$
\frac{d \tilde{l}}{d h}=\tilde{v}_{n+1}-\tilde{v}_{n} .
$$$$
\left[\begin{array}{c}
\vdots \\
l_{n, i} \\
\vdots
\end{array}\right]_{N}+\left[\begin{array}{c}
\vdots \\
d_{i} \\
\vdots
\end{array}\right]_{N}=\left[\begin{array}{c}
\vdots \\
v_{n+1, i} \\
\vdots
\end{array}\right]_{N}+\left[\begin{array}{c}
\vdots \\
f_{i} \\
\vdots
\end{array}\right]_{N}+\mathbf{A}_{N \times R} \sum_{j=1}^{n} \xi_{j, \mathrm{ref} .}
$$

In a reactive rectifier, the operation line equation can be written as follows:

Applying the same derivation as before, the following transformed variables (A.13) are introduced:

$$
\begin{gathered}
\tilde{\mathbf{d}} \equiv\left[\begin{array}{c}
\vdots \\
\tilde{d} \\
\vdots
\end{array}\right]_{N}=\left[\begin{array}{c}
\vdots \\
d_{i} \\
\vdots
\end{array}\right]_{N}-\mathbf{A}_{N \times R} \mathbf{B}_{N \text { ref } \times R}^{-1}\left[\begin{array}{c}
\vdots \\
d_{\mathrm{ref}} \\
\vdots
\end{array}\right]_{N \text { ref }}, \\
\tilde{\mathbf{f}}_{n} \equiv\left[\begin{array}{c}
\vdots \\
\tilde{f}_{n} \\
\vdots
\end{array}\right]_{N}=\left[\begin{array}{c}
\vdots \\
f_{n, i} \\
\vdots
\end{array}\right]_{N}-\mathbf{A}_{N \times R} \mathbf{B}_{N \text { ref } \times R}^{-1}\left[\begin{array}{c}
\vdots \\
f_{n, \text { ref }} \\
\vdots
\end{array}\right]_{N \text { ref }} .
\end{gathered}
$$

The reactive operation line equation can be written using these transformed variables:

$$
\tilde{v}_{n+1}=\tilde{d}+\tilde{l}_{n}-\tilde{f} .
$$

\section{B. Appendix B}

Appendix B is devoted to present the derivation of the still path of a batch middle-vessel column. This derivation applies the same principles as by Stéger et al. [2] but for multireaction systems and in consideration with additional feeds.

The differential component balance of the middle vessel with any number of reactions can be written as follows:

$$
\begin{aligned}
\frac{d\left(\mathbf{U}_{M V} \mathbf{x}_{M V}\right)}{d t}= & -D \mathbf{x}_{D}-W \mathbf{x}_{W}+\mathbf{A}_{N \times R} \sum_{\text {column }} \dot{\xi} \\
& +F_{d} \mathbf{x}_{d f}+F_{w} \mathbf{x}_{w f} .
\end{aligned}
$$


The reaction term can be eliminated with the application of a reference component

$$
\begin{aligned}
& \sum_{\text {column }} \dot{\xi} \\
& =\mathbf{B}_{R}^{-1}\left(D \mathbf{x}_{D, \text { ref }}+W \mathbf{x}_{W, \text { ref }}+\frac{d\left(U_{M V} \mathbf{x}_{M V, \text { ref }}\right)}{d t}\right. \\
& \left.-F_{d} \mathbf{x}_{d f \text { ref }}-F_{w} \mathbf{x}_{w f \text { ref }}\right), \\
& \frac{d\left(U_{M V} \mathbf{x}_{M V}\right)}{d t}-\mathbf{A}_{N \times R} \mathbf{B}_{R \times R}^{-1} \frac{d\left(U_{M V} \mathbf{x}_{M V, \mathrm{ref}}\right)}{d t} \\
& =-D\left(\mathbf{x}_{D}-\mathbf{A}_{N \times R} \mathbf{B}_{R \times R}^{-1} \mathbf{x}_{D, \text { ref }}\right) \\
& -W\left(\mathbf{x}_{W}-\mathbf{A}_{N \times R} \mathbf{B}_{R \times R}^{-1} \mathbf{x}_{W, \text { ref }}\right) \\
& +F_{d}\left(\mathbf{x}_{d f}-\mathbf{A}_{N \times R} \mathbf{B}_{R \times R}^{-1} \mathbf{x}_{d f \text { ref }}\right) \\
& +F_{w}\left(\mathbf{x}_{w f}-\mathbf{A}_{N \times R} \mathbf{B}_{R \times R}^{-1} \mathbf{x}_{w f \text { ref }}\right) .
\end{aligned}
$$

The right-hand side of the equation can be written in a simpler form with the help of the transformed variables suggested by $[5,15,16]$ as follows:

$$
\begin{aligned}
& \frac{d\left(U_{M V} \mathbf{x}_{M V}\right)}{d t}-\mathbf{A}_{N \times R} \mathbf{B}_{R \times R}^{-1} \frac{d\left(U_{M V} \mathbf{x}_{M V, \mathrm{ref}}\right)}{d t} \\
& =-D\left(1-\nu_{\mathrm{T} 1 \times R} \mathbf{B}_{R \times R}^{-1} \mathbf{x}_{D, \mathrm{ref}}\right) \frac{\mathbf{x}_{D, i}-\mathbf{A}_{N \times R} \mathbf{B}_{R \times R}^{-1} \mathbf{x}_{D, \mathrm{ref}}}{1-\nu_{\mathrm{T} 1 \times R} \mathbf{B}_{R \times R}^{-1} \mathbf{x}_{D, \mathrm{ref}}} \\
& \quad-W\left(1-\nu_{\mathrm{T} 1 \times R} \mathbf{B}_{R \times R}^{-1} \mathbf{x}_{W, \mathrm{ref}}\right) \frac{\mathbf{x}_{W, i}-\mathbf{A}_{N \times R} \mathbf{B}_{R \times R}^{-1} \mathbf{x}_{W, \mathrm{ref}}}{1-\nu_{\mathrm{T} 1 \times R} \mathbf{B}_{R \times R}^{-1} x_{W, \mathrm{ref}}} \\
& +F_{d}\left(1-\nu_{\mathrm{T} 1 \times R} \mathbf{B}_{R \times R}^{-1} \mathbf{x}_{d f, \mathrm{ref}}\right) \frac{\mathbf{x}_{d f, i}-\mathbf{A}_{N \times R} \mathbf{B}_{R \times R}^{-1} \mathbf{x}_{d f, \mathrm{ref}}}{1-\nu_{\mathrm{T} 1 \times R} \mathbf{B}_{R \times R}^{-1} \mathbf{x}_{d f, \mathrm{ref}}} \\
& \quad+F_{w}\left(1-\nu_{\mathrm{T} 1 \times R} \mathbf{B}_{R \times R}^{-1} \mathbf{x}_{w f, \mathrm{ref}}\right) \frac{\mathbf{x}_{w f, i}-\mathbf{A}_{N \times R} \mathbf{B}_{R \times R}^{-1} \mathbf{x}_{w f, \mathrm{ref}}}{1-\nu_{\mathrm{T} 1 \times R} \mathbf{B}_{R \times R}^{-1} \mathbf{x}_{w f, \mathrm{ref}}} \\
& \frac{d\left(U_{M V} \mathbf{x}_{M V, i}\right)}{d t}-\mathbf{A}_{N \times R} \mathbf{B}_{R \times R}^{-1} \frac{d\left(U_{M V} \mathbf{x}_{M V, \mathrm{ref}}\right)}{d t} \\
& =-\hat{D} \mathbf{X}_{D, i}-\widehat{W} \mathbf{X}_{W, i}+\widehat{F}_{d} \mathbf{X}_{d f, i}+\widehat{F}_{w} \mathbf{X}_{w f, i} .
\end{aligned}
$$

The left-hand side of the equation changes as follows:

$$
\begin{aligned}
& \frac{d\left(U_{M V} \mathbf{x}_{M V}\right)}{d t}-\mathbf{A}_{N \times R} \mathbf{B}_{R \times R}^{-1} \frac{d\left(U_{M V} \mathbf{x}_{M V, \mathrm{ref}}\right)}{d t} \\
& =U_{M V} \frac{d \mathbf{x}_{M V}}{d t}+\mathbf{x}_{M V} \frac{d U_{M V}}{d t}-\mathbf{A}_{N \times R} \mathbf{B}_{R \times R}^{-1} U_{M V} \frac{d \mathbf{x}_{M V, \mathrm{ref}}}{d t} \\
& \quad-\mathbf{A}_{N \times R} \mathbf{B}_{R \times R}^{-1} \mathbf{x}_{M V, \text { ref }} \frac{d U_{M V}}{d t}, \\
& \frac{d\left(U_{M V} \mathbf{x}_{M V}\right)}{d t}-\mathbf{A}_{N \times R} \mathbf{B}_{R \times R}^{-1} \frac{d\left(U_{M V} \mathbf{x}_{M V, \mathrm{ref}}\right)}{d t} \\
& =U_{M V}\left(\frac{d \mathbf{x}_{M V, i}}{d t}-\mathbf{A}_{N \times R} \mathbf{B}_{R \times R}^{-1} \frac{\left.d \mathbf{x}_{M V, \mathrm{ref}}\right)}{d t}\right) \\
& +\mathbf{X}_{M V, i}\left(1-\mathbf{A}_{N \times R} \mathbf{B}_{R \times R}^{-1} \mathbf{x}_{M V, \mathrm{ref}}\right) \mathbf{X}_{M V, i} \frac{d U_{M V}}{d t},
\end{aligned}
$$

where the $d \mathbf{X}_{M V, i} / d t$ term can be expressed with the differential form of the reactive mole fraction:

$$
\frac{d \mathbf{X}_{M V, i}}{d t}=\frac{d(\alpha / \beta)}{d t}
$$

where

$$
\begin{gathered}
\alpha=\mathbf{x}_{M V, i}-\mathbf{A}_{N x R} \mathbf{B}_{R x R}^{-1} \mathbf{x}_{M V, r e f} \\
\beta=1-\nu_{\mathrm{T} 1 x R} \mathbf{B}_{R x R}^{-1} \mathbf{x}_{M V, r e f} \\
\frac{d \alpha}{d t}=\frac{d \mathbf{x}_{M V, i}}{d t}-\mathbf{A}_{N x R} \mathbf{B}_{R x R}^{-1} \frac{d \mathbf{x}_{M V, r e f}}{d t} \\
\frac{d \beta}{d t}=-\nu_{\mathrm{T} 1 x R} \mathbf{B}_{R x R}^{-1} \frac{d \mathbf{x}_{M V, r e f}}{d t} \\
\frac{d \mathbf{X}_{M V, i}}{d t}=\frac{d \alpha / d t \cdot \beta-d \beta / d t \alpha}{\beta^{2}}
\end{gathered}
$$

where $\mathfrak{T}$ denotes $\left(\left(d \mathbf{x}_{M V, i} / d t\right)-\mathbf{A}_{N \times R} \mathbf{B}_{R \times R}^{-1}\left(d \mathbf{x}_{M V, \text { ref }} / d t\right)\right)-$ $\left(\mathbf{x}_{M V, i}-\mathbf{A}_{N \times R} \mathbf{B}_{R \times R}^{-1} \mathbf{x}_{M V, \text { ref }}\right)\left(-\nu_{\mathrm{T} 1 \times R} \mathbf{B}_{R \times R}^{-1}\left(d \mathbf{x}_{M V, \text { ref }} / d t\right)\right)$,

$$
\begin{aligned}
& \frac{d \mathbf{x}_{M V}}{d t}-\mathbf{A}_{N \times R} \mathbf{B}_{R \times R}^{-1} \frac{d \mathbf{x}_{M V, \text { ref }}}{d t} \\
& =\frac{\mathfrak{Z}}{\left(1-\mathbf{B}_{R \times R}^{-1} \nu_{\mathrm{T} 1 \times R} \mathbf{x}_{M V, \text { ref }}\right)},
\end{aligned}
$$

where $\mathfrak{Z}$ denotes $\left(d \mathbf{X}_{M V, i} / d t\right)\left(1-\mathbf{B}_{R \times R}^{-1} \nu_{\mathrm{T} 1 \times R} \mathbf{x}_{M V \text {,ref }}\right)^{2}$ $+\left(\mathbf{x}_{M V, i}-\mathbf{A}_{N \times R} \mathbf{B}_{R \times R}^{-1} \mathbf{x}_{M V, \text { ref }}\right)\left(-\nu_{\mathrm{T} 1 \times R} \mathbf{B}_{R \times R}^{-1}\left(d \mathbf{x}_{M V, \text { ref }} / d t\right)\right)$,

$$
\begin{aligned}
& \frac{d \mathbf{x}_{M V}}{d t}-\mathbf{A}_{N \times R} \mathbf{B}_{R \times R}^{-1} \frac{d \mathbf{x}_{M V, \text { ref }}}{d t} \\
& =\frac{d \mathbf{X}_{M V}}{d t}\left(1-\nu_{\mathrm{T} 1 \times R} \mathbf{B}_{R \times R}^{-1} \mathbf{x}_{M V, \text { ref }}\right) \\
& -\mathbf{X}_{M V, i} \nu_{\mathrm{T} 1 \times R} \mathbf{B}_{R \times R}^{-1} \frac{d \mathbf{x}_{M V, \text { ref }}}{d t} .
\end{aligned}
$$


Equation (B.8) can be substituted into the term of the first bracket on the right side of (B.4) as follows:

$$
\begin{aligned}
& \frac{d\left(U_{M V} \mathbf{x}_{M V}\right)}{d t}-\mathbf{A}_{N \times R} \mathbf{B}_{R \times R}^{-1} \frac{d\left(U_{M V} \mathbf{x}_{M V, \text { ref }}\right)}{d t} \\
& =\hat{U}_{M V} \frac{d \mathbf{X}_{M V}}{d t}+\mathbf{X}_{M V, i}\left(1-\nu_{\mathrm{T} 1 \times R} \mathbf{B}_{R \times R}^{-1} \mathbf{x}_{M V, \text { ref }}\right) \frac{d U_{M V}}{d t} \\
& \quad-U_{M V} \mathbf{X}_{M V} \nu_{\mathrm{T} 1 \times R} \mathbf{B}_{R \times R}^{-1} \frac{d \mathbf{x}_{M V, \text { ref }}}{d t}
\end{aligned}
$$

Equations (B.2) and (B.9) have the same terms on the left side, thus they are equal.

$$
\begin{aligned}
& \hat{U}_{M V} \frac{d \mathbf{X}_{M V}}{d t} \\
& =-\mathbf{X}_{M V}\left(1-\nu_{\mathbf{T} 1 \times R} \mathbf{B}_{R \times R}^{-1} \mathbf{x}_{M V, \text { ref }}\right) \frac{d U_{M V}}{d t} \\
& +U_{M V} \mathbf{X}_{M V, i} \nu_{\mathrm{T} 1 \times R} \mathbf{B}_{R \times R}^{-1} \frac{d \mathbf{x}_{M V, \text { ref }}}{d t} \\
& -\hat{D} \mathbf{X}_{D, i}-\widehat{W} \mathbf{X}_{W, i}+\widehat{F}_{d} \mathbf{X}_{d f, i}+\widehat{F}_{w} \mathbf{X}_{w f, i} .
\end{aligned}
$$

The total mass balance of the middle vessel is defined as follows:

$$
\frac{d U_{M V}}{d t}=-D-W+\nu_{T} \sum_{\text {column }} \dot{\xi}+F_{d}+F_{w} .
$$

Substituting (B.11) into (B.10) gives the final form of the general equation of the still path in the presence of more instantaneously equilibrium limited reactions.

$$
\begin{aligned}
\hat{U}_{M V} \frac{d \mathbf{X}_{M V}}{d t}= & -\mathbf{X}_{M V}\left(1-\nu_{\mathrm{T} 1 \times R} \mathbf{B}_{R \times R}^{-1} \mathbf{X}_{M V, \text { ref }}\right) \\
& \times\left(-D-W+\nu_{\mathrm{T} 1 \times R} \sum_{\text {column }} \dot{\xi}+F_{d}+F_{w}\right) \\
& +U_{M V} \mathbf{X}_{M V} \nu_{\mathrm{T} 1 \times R} \mathbf{B}_{R \times R}^{-1} \frac{d \mathbf{x}_{M V, \text { ref }}}{d t}-\hat{D} \mathbf{X}_{D, i} \\
& -\widehat{W} \mathbf{X}_{W, i}+\widehat{F}_{d} \mathbf{X}_{d f}+\widehat{F}_{w} \mathbf{X}_{w f},
\end{aligned}
$$

$$
\begin{aligned}
\hat{U}_{M V} \frac{d \mathbf{X}_{M V, i}}{d t}= & \hat{D}\left(\mathbf{X}_{M V, i} \frac{1-\mathbf{B}_{R \times R}^{-1} \nu_{\mathrm{T} 1 \times R} \mathbf{x}_{M V, \text { ref }}}{1-\mathbf{B}_{R \times R}^{-1} \nu_{\mathrm{T} 1 \times R} \mathbf{X}_{D, \mathrm{ref}}}-\mathbf{X}_{D, i}\right) \\
& +\widehat{W}\left(\mathbf{X}_{M V, i} \frac{1-\mathbf{B}_{R \times R}^{-1} \nu_{\mathrm{T} 1 \times R} \mathbf{x}_{M V, \text { ref }}}{1-\mathbf{B}_{R \times R}^{-1} \nu_{\mathrm{T} 1 \times R} \mathbf{x}_{W, \text { ref }}}-\mathbf{X}_{W, i}\right) \\
& -\widehat{F}_{d}\left(\mathbf{X}_{d f, i} \frac{1-\mathbf{B}_{R \times R}^{-1} \nu_{\mathrm{T} 1 \times R} \mathbf{x}_{d f, \text { ref }}}{1-\mathbf{B}_{R \times R}^{-1} \nu_{\mathrm{T} 1 \times R} \mathbf{X}_{d f, \text { ref }}}-\mathbf{X}_{d f, i}\right) \\
& -\widehat{F}_{w}\left(\mathbf{X}_{w f, i} \frac{1-\mathbf{B}_{R \times R}^{-1} \nu_{\mathrm{T} 1 \times R} \mathbf{X}_{w f, \text { ref }}}{1-\mathbf{B}_{R \times R}^{-1} \nu_{\mathrm{T} 1 \times R} \mathbf{x}_{w f, \text { ref }}} \mathbf{X}_{w f, i}\right) \\
& -\mathbf{X}_{M V, i}\left(1-\mathbf{B}_{R \times R}^{-1} \nu_{\mathrm{T} 1 \times R} \mathbf{x}_{M V, \text { ref }}\right) \nu_{T} \sum_{\text {oszlop }} \dot{\xi} \\
& +U_{M V} \mathbf{X}_{M V, i} \mathbf{B}_{R \times R}^{-1} \nu_{\mathrm{T} 1 \times R} \frac{d \mathbf{x}_{M V, \text { ref }}}{d t} .
\end{aligned}
$$

The graphical solution of the general equation is impossible, but its form is much simpler in the case of equimolar reactions, namely, when $\nu_{T}=0$. In the case of an equimolar reaction the reactive flow rates are equal to the nonreactive ones; therefore $\widehat{U}_{M V}=U_{M V}, \widehat{D}=D$ and $\widehat{W}=W$. Therefore, in the case of equimolar reactions, the equation of the still path is the following:

$$
\begin{aligned}
\frac{d \mathbf{X}_{M V, i}}{d \tau}= & D\left(\mathbf{X}_{M V, i}-\mathbf{X}_{D, i}\right)+W\left(\mathbf{X}_{M V, i}-\mathbf{X}_{W, i}\right) \\
& -F_{d}\left(\mathbf{X}_{M V, i}-\mathbf{X}_{f d, i}\right)-F_{w}\left(\mathbf{X}_{M V, i}-\mathbf{X}_{f w, i}\right) .
\end{aligned}
$$

\section{Abbreviations}

A: Matrix of stoichiometric coefficients of the components in the reactions

B: Matrix of stoichiometric coefficients of the reference components

$D$ : Distillate flow rate $(\mathrm{mol} / \mathrm{s})$

$d$ : Distillate component flow rate $(\mathrm{mol} / \mathrm{s})$

$F:$ Feed flow rate $(\mathrm{mol} / \mathrm{s})$

$f$ : Feed component flow rate $(\mathrm{mol} / \mathrm{s})$

$h$ : Dimensionless height

$K$ : Equilibrium constant expressed in concentration

$l$ : Liquid component flow rate $(\mathrm{mol} / \mathrm{s})$

$L$ : Molar liquid flow rate $(\mathrm{mol} / \mathrm{s})$

$p_{i}^{o}:$ Vapor pressure $(\mathrm{mmHg}=133.322 \mathrm{~Pa})$

$T$ : Temperature $(\mathrm{K})$

$U$ : Molar holdup in the still (mol)

$V:$ Molar vapor flow rate $(\mathrm{mol} / \mathrm{s})$

$W$ : Molar flow rate of bottom product ( $\mathrm{mol} / \mathrm{s}$ )

$w$ : Component flow rate of bottom product $(\mathrm{mol} / \mathrm{s})$

$v$ : Vapor component flow rate $(\mathrm{mol} / \mathrm{s})$

$x$ : Liquid composition (mole fractions)

$X$ : Transformed composition (mole fraction)

$Y$ : Vapor composition (mole fractions) 
$y^{*}$ : Vapor composition (mole fractions) in equilibrium with $x$

$z$ : Feed composition (mole fractions)

$v$ : Stoichiometric coefficient

$\xi$ : Reaction coordinate $(\mathrm{mol} / \mathrm{s})$.

\section{Subscripts and Superscripts}

D: Distillate

$d f$ : Feed in the upper section of the middle-vessel column

$i$ : $\quad$ ith component

$M V$ : Middle vessel

Nc: Number of components

$R: \quad$ Number of reference components

ref: Reference component

T: total

$W$ : Bottom product

$w f$ : Feed in the lower section of the middle vessel column

： Transformed variable.

\section{Acknowledgments}

The research work in the present paper was supported by the Hungarian and French Government and by the Hungarian Fund of OTKA K062099.

\section{References}

[1] M. F. Malone and M. F. Doherty, "Reactive distillation," Industrial and Engineering Chemistry Research, vol. 39, no. 11, pp. 3953-3957, 2000.

[2] C. Stéger, T. Lukács, E. Rév, M. Meyer, and Z. Lelkes, "An overall feasibility study of batch reactive distillation in hybrid configurations," AIChE Journal, vol. 55, no. 5, pp. 1185-1199, 2009.

[3] D. Barbosa and M. F. Doherty, "Design and minimum-reflux calculations for single-feed multicomponent reactive distillation columns," Chemical Engineering Science, vol. 43, no. 7, pp. 1523-1537, 1988.

[4] J. Espinosa, "On the integration of reaction and separation in a batch extractive distillation column with a middle vessel," Industrial and Engineering Chemistry Research, vol. 41, no. 15, pp. 3657-3668, 2002.

[5] J. Espinosa, P. A. Aguirre, and G. A. Perez, "Product composition regions of single-feed reactive distillation columns: mixtures containing inerts," Industrial and Engineering Chemistry Research, vol. 34, no. 3, pp. 853-861, 1995.

[6] J. Espinosa, P. Aguirre, and G. Perez, "Some Some aspects in the design of multicomponent reactive distillation columns with a reacting core: mixtures containing inerts," Industrial and Engineering Chemistry Research, vol. 35, no. 12, pp. 45374549, 1996.

[7] R. M. Dragomir and M. Jobson, "Conceptual design of singlefeed hybrid reactive distillation columns," Chemical Engineering Science, vol. 60, no. 16, pp. 4377-4395, 2005.

[8] C. Stéger, R. Thery, X. Meyer, M. Meyer, M. Brehelin, and D. Amoros, "Étude de faisabilité de l'estérification d'un diacide par distillation réactive," Oil \& Gas Science and TechnologyRevue d'IFP Energies nouvelles, vol. 65, no. 5, pp. 703-719, 2010.

[9] H.-P. Luo and W.-D. Xiao, "A reactive distillation process for a cascade and azeotropic reaction system: carbonylation of ethanol with dimethyl carbonate," Chemical Engineering Science, vol. 56, no. 2, pp. 403-410, 2001.

[10] Z. Lelkes, P. Lang, B. Benadda, and P. Moszkowicz, "Feasibility of extractive distillation in a batch rectifier," AIChE Journal, vol. 44, no. 4, pp. 810-822, 1998.

[11] C. Stéger, V. Varga, L. Horváth et al., "Feasibility of extractive distillation process variants in batch rectifier column," Chemical Engineering and Processing, vol. 44, no. 11, pp. 1237-1256, 2005.

[12] V. Varga, E. R. Frits, and V. Gerbaud, "Separation of azeotropes in batch extractive stripper with intermediate entrainer," in Proceedings of the 16th European Symposium on Computer Aided Process Engineering, (ESCAPE '06) and 9th International Symposium on Process Systems Engineering, (PSE '06), Garmisch-Partenkirchen, Germany, July 2006.

[13] C. Stéger, E. Rév, L. Horváth, Z. Fonyó, M. Meyer, and Z. Lelkes, "New extractive configuration separating azeotropic mixture in semi-batch way," Separation and Purification Technology, vol. 52, no. 2, pp. 343-356, 2006.

[14] M. Doherty and G. Buzad, "Reactive distillation by design," Transactions of the Institution of Chemical Engineers, vol. 90, no. A, pp. 448-458, 1992.

[15] H.-P. Luo, W.-D. Xiao, and K.-H. Zhu, "Isobaric vapor-liquid equilibria of alkyl carbonates with alcohols," Fluid Phase Equilibria, vol. 175, no. 1-2, pp. 91-105, 2000.

[16] K. Sundmacher and A. Kienle, Eds., Reactive Distillation, Wiley-VCH, Weinheim, Germany, 2002. 

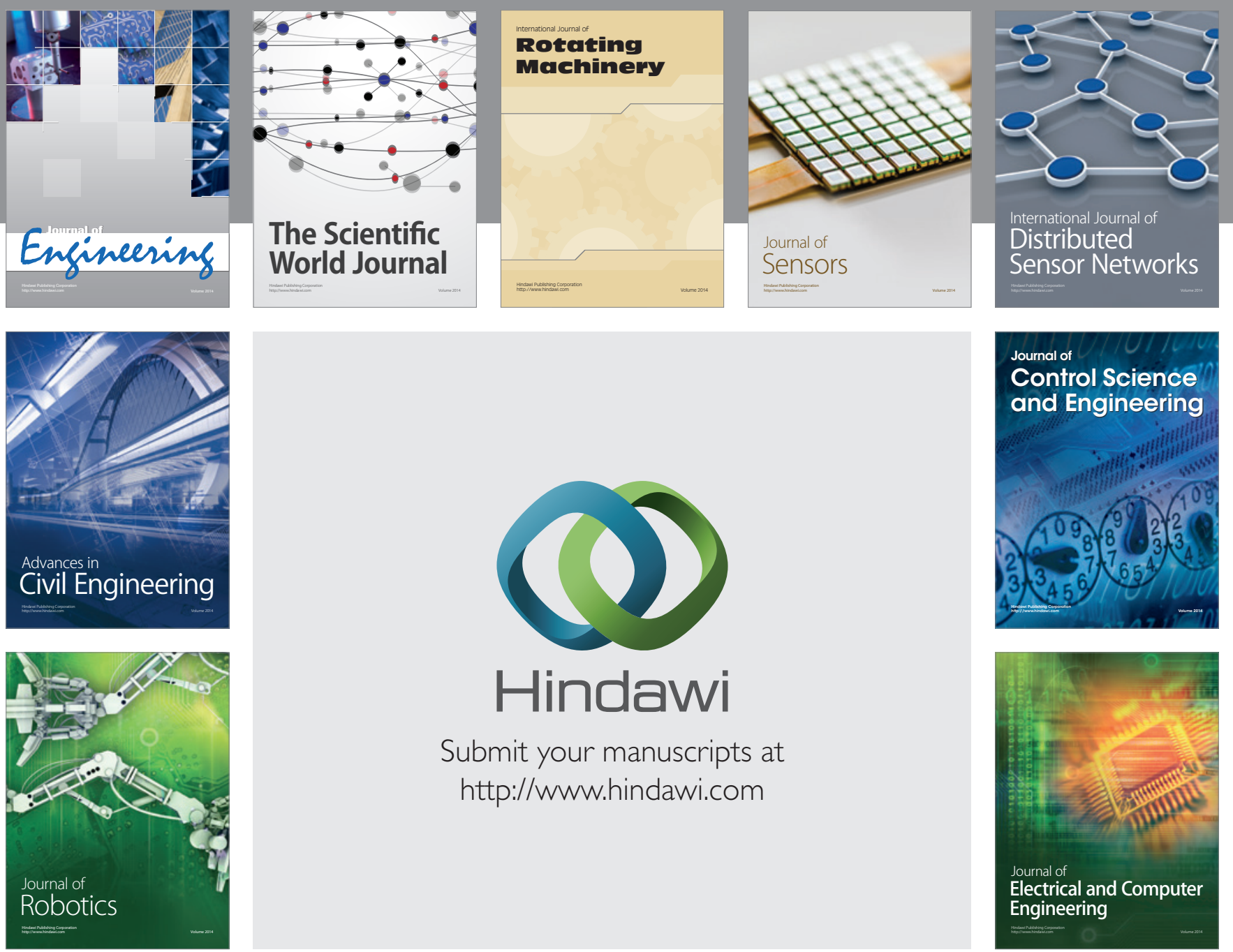

Submit your manuscripts at

http://www.hindawi.com
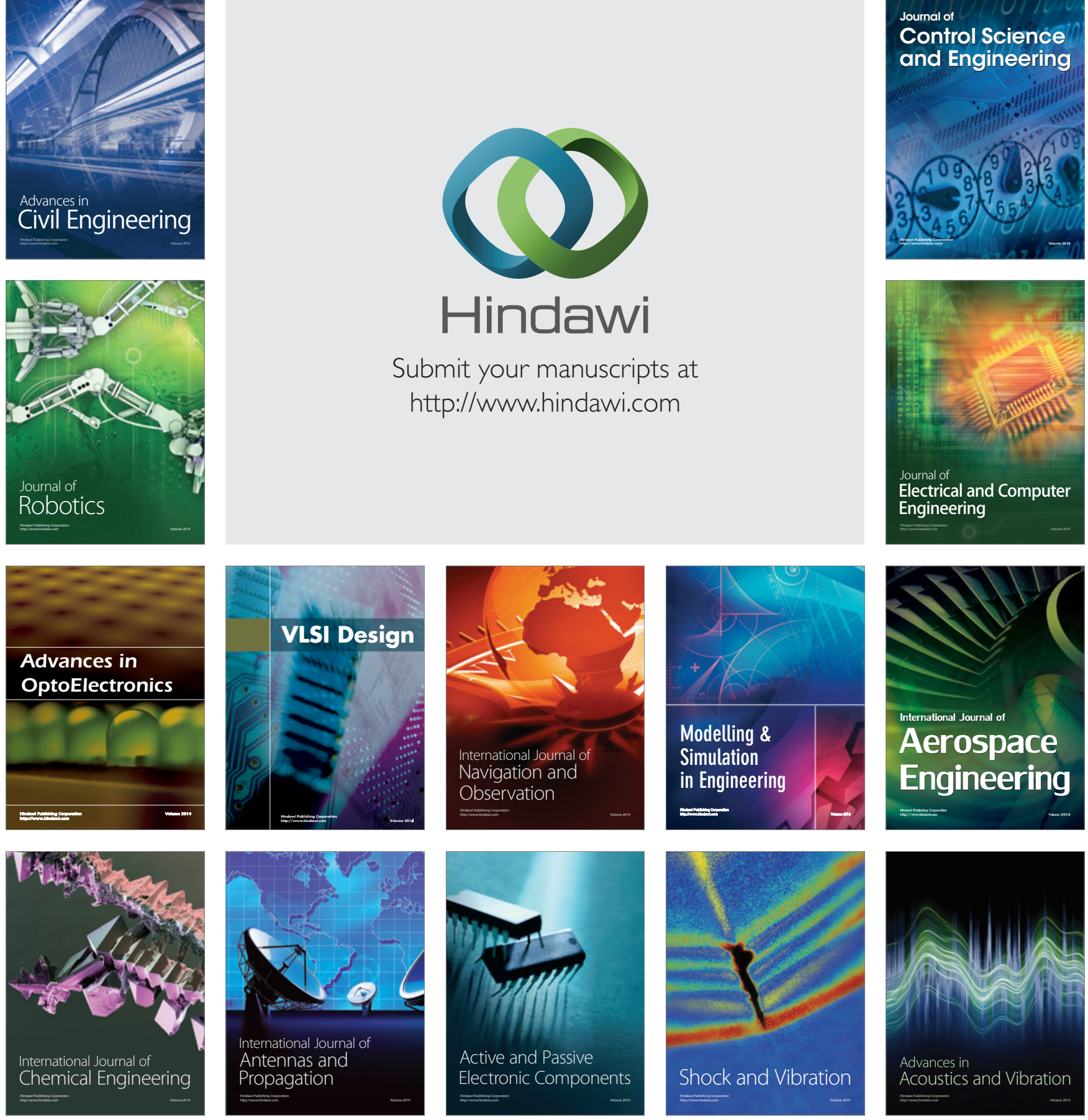